\begin{tabular}{|c|l|}
\hline Title & Openings of differentiable map-germs and unfoldings \\
\hline Author(s) & Ishikawa, Goo \\
\hline Citation & Hokkaido University Preprint Series in Mathematics, 1016, 1-26 \\
\hline Issue Date & 2012-8-20 \\
\hline DOI & 10.14943/84162 \\
\hline Doc URL & http://hdl.handle.net/2115/69821 \\
\hline Type & bulletin (article) \\
\hline File Information & pre1016.pdf \\
\hline
\end{tabular}

Instructions for use 


\title{
Openings of differentiable map-germs and unfoldings
}

\author{
Goo Ishikawa
}

Dedicated to Professor Satoshi Koike for his 60th birthday

\begin{abstract}
The algebraic notion of openings of a map-germ is introduced in this paper. An opening separates the self-intersections of the original mapgerm, preserving its singularities. The notion of openings is different from the notion of unfoldings. Openings do not unfold the singularities. For example, the swallowtail is an opening of the Whitney's cusp mapgerm from plane to plane and the open swallowtail is a versal opening of them. Openings of map-germs appear as typical singularities in several problems of geometry and its applications. The notion of openings has close relations to isotropic map-germs in a symplectic space and integral map-germs in a contact space. We describe the openings of Morin singularities, namely, stable unfoldings of map-germs of corank one. The relation of unfoldings and openings are discussed. Moreover we provide a method to construct versal openings of map-germs and give versal openings of stable map-germs $\left(\mathbf{R}^{4}, 0\right) \rightarrow\left(\mathbf{R}^{4}, 0\right)$. Lastly the relation of lowerable vector fields and openings is discussed.
\end{abstract}

\section{Introduction}

There is a sequence of well-known singularities of map-germs: The Whitney's cusp $f:\left(\mathbf{R}^{2}, 0\right) \rightarrow\left(\mathbf{R}^{2}, 0\right), f(x, u)=\left(x^{3}+u x, u\right)$, the swallowtail $F:$ $\left(\mathbf{R}^{2}, 0\right) \rightarrow\left(\mathbf{R}^{3}, 0\right), F(x, u)=\left(f(x, u), x^{4}+\frac{2}{3} u x^{2}\right)$, and the open swallowtail $\widetilde{F}:\left(\mathbf{R}^{2}, 0\right) \rightarrow\left(\mathbf{R}^{4}, 0\right), \widetilde{F}(x, u)=\left(F(x, u), x^{5}+\frac{4}{9} u x^{3}\right)$.

They have the same singular locus and the same kernel field of the differential along the singular locus, while the self-intersections are resolved.

What is the algebraic structure behind them? One of answers to the above question is presented in this paper. In fact we observe, for the swal- 

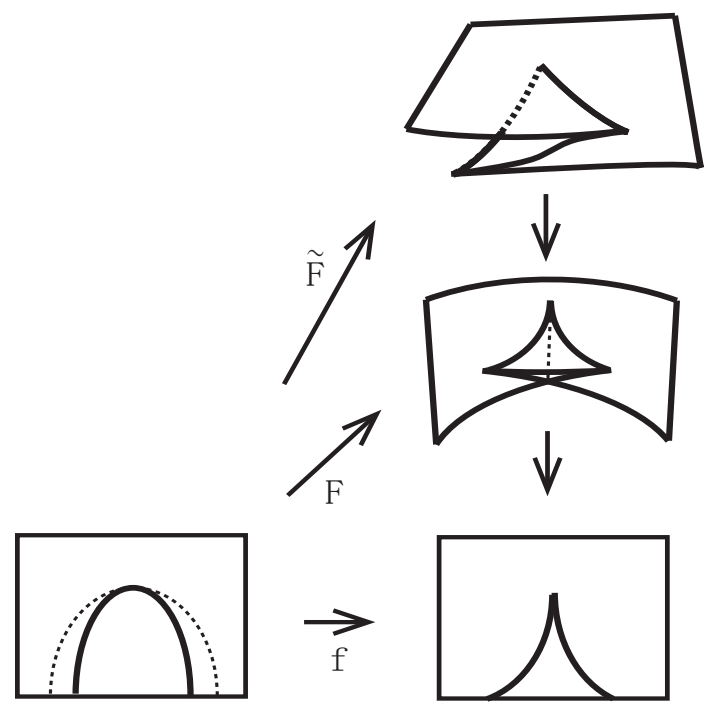

lowtail $F(x, u)=\left(x^{3}+u x, u, x^{4}+\frac{2}{3} u x^{2}\right)$, we see that

$$
d\left(x^{4}+\frac{2}{3} u x^{2}\right)=\frac{4}{3} x d\left(x^{3}+u x\right)-\frac{4}{9} x^{2} d u \in\left\langle d\left(x^{3}+u x\right), d u\right\rangle_{\mathcal{E}_{2}} .
$$

For the open swallowtail $\widetilde{F}(x, u)=\left(x^{3}+u x, u, x^{4}+\frac{2}{3} u x^{2}, x^{5}+\frac{4}{9} u x^{3}\right)$, we have that

$$
d\left(x^{5}+\frac{4}{9} u x^{3}\right)=\frac{5}{3} x^{2} d\left(x^{3}+u x\right)-\frac{10}{9} x^{3} d u \in\left\langle d\left(x^{3}+u x\right), d u\right\rangle_{\mathcal{E}_{2}} .
$$

Here $d$ means the exterior differential and $\mathcal{E}_{2}$ denotes the $\mathbf{R}$-algebra of $C^{\infty}$ function-germs on $\left(\mathbf{R}^{2}, 0\right)$.

As the key construction, we introduce the notion of opening of multigerms of mappings. To do this, first we recall the auxiliary notions in this paper.

Let $f:\left(\mathbf{R}^{n}, A\right) \rightarrow\left(\mathbf{R}^{m}, b\right)$ be a multi-germ of a $C^{\infty}$ map with $n \leq m$. Here $A$ is a finite subset of $\mathbf{R}^{n}, b \in \mathbf{R}^{m}$ and $f(A)=\{b\}$. We define the Jacobi module $\mathcal{J}_{f}$ of $f$ by

$$
\mathcal{J}_{f}=\left\{\sum_{j=1}^{m} p_{j} d f_{j} \mid p_{j} \in \mathcal{E}_{\mathbf{R}^{n}, A}(1 \leq j \leq m)\right\} \subset \Omega_{\mathbf{R}^{n}, A}^{1}
$$

in the space $\Omega_{\mathbf{R}^{n}, A}^{1}$ of 1 -form-germs on $\left(\mathbf{R}^{n}, A\right)$. Note that $\mathcal{J}_{f}$ is just the first order component of the graded differential ideal $\mathcal{J}_{f}^{\bullet}$ in $\Omega_{\mathbf{R}^{n}, A}^{\bullet}$ generated 
by $d f_{1}, \ldots, d f_{m}$. Then the singular locus, the non-immersive locus, of $f$ is given by

$$
\Sigma_{f}=\left\{x \in\left(\mathbf{R}^{n}, A\right) \mid \operatorname{rank} \mathcal{J}_{f}(x)<n\right\} .
$$

Also we consider the kernel field $\operatorname{Ker}\left(f_{*}: T \mathbf{R}^{n} \rightarrow T \mathbf{R}^{m}\right)$ of the differential of $f$, along $\Sigma_{f}$.

For another map-germ $f^{\prime}:\left(\mathbf{R}^{n}, A\right) \rightarrow\left(\mathbf{R}^{m^{\prime}}, b^{\prime}\right), n \leq m^{\prime}$, if $\mathcal{J}_{f^{\prime}}=\mathcal{J}_{f}$, then $\Sigma_{f^{\prime}}=\Sigma_{f}$ and $\operatorname{Ker}\left(f_{*}^{\prime}\right)=\operatorname{Ker}\left(f_{*}\right)$.

Then define the ramification module $\mathcal{R}_{f}$ of $f$ by

$$
\mathcal{R}_{f}=\left\{h \in \mathcal{E}_{\mathbf{R}^{n}, A} \mid d h \in \mathcal{J}_{f}\right\},
$$

(cf. ([11][14]). Remark that a related notion was introduced in [22] from different motivation.

For $f:\left(\mathbf{R}^{n}, A\right) \rightarrow\left(\mathbf{R}^{m}, b\right), f^{\prime}:\left(\mathbf{R}^{n}, A\right) \rightarrow\left(\mathbf{R}^{m^{\prime}}, b^{\prime}\right)$, easily we see that $\mathcal{J}_{f^{\prime}}=\mathcal{J}_{f}$ if and only if $\mathcal{R}_{f^{\prime}}=\mathcal{R}_{f}$ (Lemma 2.1 ).

Definition 1.1 Given $h_{1}, \ldots, h_{r} \in \mathcal{R}_{f}$, the map-germ $F:\left(\mathbf{R}^{n}, A\right) \rightarrow \mathbf{R}^{m} \times$ $\mathbf{R}^{r}=\mathbf{R}^{m+r}$ defined by

$$
F=\left(f_{1}, \ldots, f_{m}, h_{1}, \ldots, h_{r}\right)
$$

is called an opening of $f$, while $f$ is called a closing of $F$.

Then, for any opening $F$ of $f$, we have $\mathcal{R}_{F}=\mathcal{R}_{f}, \mathcal{J}_{F}=\mathcal{J}_{f}, \Sigma_{F}=\Sigma_{f}$ and $\operatorname{Ker}\left(F_{*}\right)=\operatorname{Ker}\left(f_{*}\right)$.

For example, the swallowtail is an opening of the Whitney's cusp. The open swallowtail is an opening of the swallowtail and of the Whitney's cusp.

Note that an opening of an opening of $f$ is an opening of $f$.

Definition 1.2 An opening $F=\left(f, h_{1}, \ldots, h_{r}\right)$ of $f$ is called a versal opening (resp. a mini-versal opening) of $f:\left(\mathbf{R}^{n}, A\right) \rightarrow\left(\mathbf{R}^{m}, b\right)$, if $1, h_{1}, \ldots, h_{r}$ form a (minimal) system of generators of $\mathcal{R}_{f}$ as an $\mathcal{E}_{\mathbf{R}^{m}, b}$-module via $f^{*}$ : $\mathcal{E}_{\mathbf{R}^{m}, b} \rightarrow \mathcal{E}_{\mathbf{R}^{n}, A}$.

Note that a versal opening of an opening of $f$ is a versal opening of $f$. An opening of a versal opening of $f$ is a versal opening of $f$.

A mini-versal opening $F:\left(\mathbf{R}^{n}, A\right) \rightarrow \mathbf{R}^{m+r}$ of $f$ is unique up to leftequivalence and a versal opening $G:\left(\mathbf{R}^{n}, A\right) \rightarrow \mathbf{R}^{m+s}$ of $f$ is left-equivalent to a mini-versal opening composed with an immersion $\left(\mathbf{R}^{n}, A\right) \rightarrow \mathbf{R}^{m+r} \hookrightarrow$ $\mathbf{R}^{m+s}$ (Proposition 2.12). 
Openings of map-germs appear as typical singularities in several problems of geometry and its applications. The openings naturally appear in the classification problem of "tangential singularities"[15][16]. Open swallowtails, open folded umbrellas, etc. appear as tangent varieties. We have applied opening constructions to solve the "stable" classification problem of tangent varieties to generic submanifolds in [16]. Moreover openings are related to singularities of isotropic mappings in symplectic spaces.

Let $T^{*} \mathbf{R}^{n}=\mathbf{R}^{2 m}$ be the $2 m$-dimensional symplectic space with the symplectic form $\omega=\sum_{i=1}^{m} d p_{i} \wedge d x_{i}$, and $f:\left(\mathbf{R}^{n}, A\right) \rightarrow \mathbf{R}^{2 m}$ a multi-germ of isotropic mapping. Since $f^{*} \omega=0$, we have that $\sum_{i=1}^{m}\left(p_{i} \circ f\right) d\left(x_{i} \circ f\right)$ is closed, so it is exact and there exists $e \in \mathcal{E}_{\mathbf{R}^{n}, A}$ such that

$$
d e=\sum_{i=1}^{m}\left(p_{i} \circ f\right) d\left(x_{i} \circ f\right) .
$$

Set $g:\left(\mathbf{R}^{n}, A\right) \rightarrow \mathbf{R}^{m}$ by $g(x)=\left(x_{1} \circ f(x), \ldots, x_{m} \circ f(x)\right)$. Then $e \in \mathcal{R}_{g}$. Conversely, given $e \in \mathcal{R}_{g}$, we have $d e=\sum_{i=1}^{m} a_{i} d g_{i}$ for some functions $a_{1}, \ldots, a_{m}$, and we obtain an isotropic multi-germ $f:\left(\mathbf{R}^{n}, A\right) \rightarrow \mathbf{R}^{2 m}$ by $p_{i} \circ f=a_{i}, x_{i} \circ f=g_{i},(1 \leq i \leq m)$. The opening $(g, e):\left(\mathbf{R}^{n}, A\right) \rightarrow \mathbf{R}^{m+1}$ is the frontal germ associated to $f$. The open folded umbrella appears also as a "frontal-symplectic singularity" [17].

In $\S 2$, we give a detailed exposition on ramification modules and openings of multi-germs and, in $\S 3$, we mention related geometric problems to motivate the subject in the present paper.

In $\S 4$, the relation of unfoldings and openings are discussed. We treat the problem to find a versal opening of an unfolding of a given map-germ. Then the notion of extendability of an unfolding is introduced. If the given mapgerm is of corank one, then any unfolding is extendable and, in particular its versal opening is obtained from that of its stable unfolding. Then, in $\S 5$, we give the explicit presentation on versal openings of stable unfoldings of map-germs of corank 1, namely, versal openings of Morin maps.

In $\S 6$, we remark the existence of versal openings in finite analytic case. In $\S 7$, we give a direct method to find versal openings for several examples and show the existence of the versal opening for any stable map-germ $\left(\mathbf{R}^{4}, A\right) \rightarrow\left(\mathbf{R}^{4}, b\right)$, explicitly. In $\S 8$, the relation of lowerable vector fields of map-germs and openings is discussed.

In this paper we often abbreviate $\mathcal{E}_{\left(\mathbf{R}^{n}, A\right)}$ by $\mathcal{E}_{A}$, the $\mathbf{R}$-algebra of $C^{\infty}$ function-germs on $\left(\mathbf{R}^{n}, A\right)$. If $A=\left\{a_{1}, \ldots, a_{s}\right\}$, then we denote by $\mathfrak{m}_{i}$ the 
maximal ideal consisting of $h \in \mathcal{E}_{A}$ with $h\left(a_{i}\right)=0$. We set $\mathfrak{m}_{A}=\cap_{i=1}^{s} \mathfrak{m}_{i}$. If $A$ consists of the origin, then we use $\mathcal{E}_{n}, \mathfrak{m}_{n}$ instead of $\mathcal{E}_{A}, \mathfrak{m}_{A}$ respectively.

All manifolds and mappings we treat in this paper are assumed to be of class $C^{\infty}$, unless otherwise stated.

\section{Ramification modules and openings}

In Introduction, we have introduced the notion of openings based on that of Jacobi modules and ramification modules.

Lemma 2.1 For map-germs $f:\left(\mathbf{R}^{n}, A\right) \rightarrow\left(\mathbf{R}^{m}, b\right), f^{\prime}:\left(\mathbf{R}^{n}, A\right) \rightarrow\left(\mathbf{R}^{m^{\prime}}, b^{\prime}\right)$, we have that $\mathcal{J}_{f^{\prime}}=\mathcal{J}_{f}$ if and only if $\mathcal{R}_{f^{\prime}}=\mathcal{R}_{f}$.

Proof: It is clear that $\mathcal{J}_{f^{\prime}}=\mathcal{J}_{f}$ implies $\mathcal{R}_{f^{\prime}}=\mathcal{R}_{f}$. Conversely suppose $\mathcal{R}_{f^{\prime}}=\mathcal{R}_{f}$. Then any component $f_{j}^{\prime}$ of $f^{\prime}$ belongs to $\mathcal{R}_{f^{\prime}}=\mathcal{R}_{f}$, hence $d f_{j}^{\prime} \in \mathcal{J}_{f}$. Therefore $\mathcal{J}_{f^{\prime}} \subseteq \mathcal{J}_{f}$. By the symmetry we have $\mathcal{J}_{f^{\prime}}=\mathcal{J}_{f}$.

Lemma 2.2 Let $f:\left(\mathbf{R}^{n}, A\right) \rightarrow\left(\mathbf{R}^{m}, b\right)$ be a map-germ. Then we have

(1) $f^{*} \mathcal{E}_{b} \subseteq \mathcal{R}_{f} \subseteq \mathcal{E}_{A}$.

(2) $\mathcal{R}_{f}$ is an $\mathcal{E}_{b}$-submodule via $f^{*}: \mathcal{E}_{b} \rightarrow \mathcal{E}_{A}$ of $\mathcal{E}_{A}$.

(3) $\mathcal{R}_{f}$ is $C^{\infty}$-subring of $\mathcal{E}_{A}$.

(4) If $\tau:\left(\mathbf{R}^{m}, b\right) \rightarrow\left(\mathbf{R}^{m}, b^{\prime}\right)$ is a diffeomorphism-germ, then $\mathcal{R}_{\tau \circ f}=\mathcal{R}_{f}$. If $\sigma:\left(\mathbf{R}^{n}, A^{\prime}\right) \rightarrow\left(\mathbf{R}^{n}, A\right)$ is a diffeomorphism-germ, then $\mathcal{R}_{f \circ \sigma}=\sigma^{*}\left(\mathcal{R}_{f}\right)$.

Proof: The assertions (1) and (2) follow from that, if $h \in \mathcal{R}_{f}$ and $d h=$ $\sum_{j=1}^{m} p_{j} d f_{j}$, then

$$
d\{(k \circ f) h\}=\sum_{j=1}^{m}\left\{(k \circ f) p_{j}+h\left(\partial k / \partial y_{j}\right)\right\} d f_{j} .
$$

The assertion (3) follows from that, if $h_{1}, \ldots, h_{r} \in \mathcal{R}_{f}$ and if $\tau: \mathbf{R}^{r} \rightarrow \mathbf{R}$ is a $C^{\infty}$ function, then

$$
d\left\{\tau\left(h_{1}, \ldots, h_{r}\right)\right\}=\sum_{i=1}^{r} \frac{\partial \tau}{\partial y_{i}}\left(h_{1}, \ldots, h_{r}\right) d h_{i} \in \mathcal{J}_{f} .
$$

The assertion (4) follows from that $\mathcal{J}_{\tau \circ f}=\mathcal{J}_{f}$ and $\mathcal{J}_{f \circ \sigma}=\sigma^{*}\left(\mathcal{J}_{f}\right)$.

Lemma 2.3 Let $f:\left(\mathbf{R}^{n}, A\right) \rightarrow\left(\mathbf{R}^{m}, b\right)$ be a map-germ with $A=\left\{a_{1}, \ldots, a_{s}\right\}$. We denote by $f_{i}:\left(\mathbf{R}^{n}, a_{i}\right) \rightarrow\left(\mathbf{R}^{m}, b\right)$ the restriction of $f$ to $\left(\mathbf{R}^{n}, a_{i}\right)$. Then $\mathcal{R}_{f} \cong \prod_{i=1}^{s} \mathcal{R}_{f_{i}}$ as $\mathcal{E}_{b}$-module. 
Proof: We have the isomorphism $\varphi: \mathcal{R}_{f} \rightarrow \prod_{i=1}^{s} \mathcal{R}_{f_{i}}$ defined by $\varphi(h)=$ $\left(\left.h\right|_{\left(\mathbf{R}^{n}, a_{i}\right)}\right)_{i=1}^{s}$.

Remark 2.4 Any multi-germ $f:\left(\mathbf{R}^{n}, A\right) \rightarrow\left(\mathbf{R}^{m}, b\right)$, \# $(A)=s$, is rightequivalent to a map-germ of form $\coprod_{s} f_{i}: \coprod_{s}\left(\mathbf{R}^{n}, 0\right) \rightarrow\left(\mathbf{R}^{m}, b\right)$ from the disjoint union of $s$-copies of $\left(\mathbf{R}^{n}, 0\right)$.

A map-germ $f:\left(\mathbf{R}^{n}, A\right) \rightarrow\left(\mathbf{R}^{m}, b\right)$ is called finite if $\mathcal{E}_{A}$ is a finite $\mathcal{E}_{b^{-}}$ module. The condition is equivalent to that $\operatorname{dim}_{\mathbf{R}} \mathcal{E}_{A} /\left(f^{*} \mathfrak{m}_{b}\right) \mathcal{E}_{A}<\infty$ by Nakayama's lemma (see for example [4]). Moreover $f$ is finite if and only if $\mathcal{K}$-finite $([23])$.

Proposition 2.5 If $f:\left(\mathbf{R}^{n}, A\right) \rightarrow\left(\mathbf{R}^{m}, b\right)$ is finite and of corank at most one. Then we have

(1) $\mathcal{R}_{f}$ is a finite $\mathcal{E}_{b}$-module. Therefore there exists a versal opening of $f$.

(2) $1, h_{1}, \ldots, h_{r} \in \mathcal{R}_{f}$ generate $\mathcal{R}_{f}$ as $\mathcal{E}_{b}$-module if and only if they generate the vector space $\mathcal{R}_{f} /\left(f^{*} \mathfrak{m}_{b}\right) \mathcal{R}_{f}$ over $\mathbf{R}$.

Proof: In the case $A$ consists of a point, the assertions are proved in Theorem 1.3 of [12] and Corollary 2.4 of [14]. For a general finite set $A$, the assertions are reduced to the case that $A$ consists of a point by Lemma 2.3 .

For a map-germ $f:\left(\mathbf{R}^{n}, A\right) \rightarrow\left(\mathbf{R}^{m}, b\right), n \leq m$, we have defined in Introduction the notions of openings and versal openings of $f$.

Example 2.6 (1) Let $h:(\mathbf{R}, 0) \rightarrow(\mathbf{R}, 0), h(x)=x^{2}$. Then $\mathcal{R}_{h}=$ $\left\langle 1, x^{3}\right\rangle_{f^{*}\left(\mathcal{E}_{1}\right)}$. The map-germ $H:(\mathbf{R}, 0) \rightarrow\left(\mathbf{R}^{2}, 0\right), H(x):=\left(x^{2}, x^{3}\right)$, the simple cusp map, is the mini-versal opening of $h$.

(2) Let $g:(\mathbf{R}, 0) \rightarrow(\mathbf{R}, 0), g(x)=x^{3}$. Then $\mathcal{R}_{g}=\left\langle 1, x^{4}, x^{5}\right\rangle_{f^{*}\left(\mathcal{E}_{1}\right)}$. The map-germ $G:(\mathbf{R}, 0) \rightarrow\left(\mathbf{R}^{3}, 0\right), G(x):=\left(x^{3}, x^{4}, x^{5}\right)$ is the mini-versal opening of $g$.

(3) Let $f:\left(\mathbf{R}^{2}, 0\right) \rightarrow\left(\mathbf{R}^{2}, 0\right), f(x, u)=\left(x^{3}+u x, u\right)$, an unfolding of $g$. Then $\mathcal{R}_{f}=\left\langle 1, x^{4}+\frac{2}{23} u x^{2}, x^{5}+\frac{4}{9} u x^{3}\right\rangle_{f^{*}\left(\mathcal{E}_{2}\right)}$. The map-germ $F:\left(\mathbf{R}^{2}, 0\right) \rightarrow$ $\left(\mathbf{R}^{4}, 0\right)$ defined by $F(x, u):=\left(x^{3}+u x, u, x^{4}+\frac{2}{23} u x^{2}, x^{5}+\frac{4}{9} u x^{3}\right)$, the open swallowtail is the mini-versal opening of Whitney's cusp $f$.

(4) Let consider the multi-germ $k:(\mathbf{R}, A) \rightarrow\left(\mathbf{R}^{2}, 0\right), A=\{0,1\}$ defined by $k(t)=(t, 0)$ near $t=0$ and $k(t)=(0, t-1)$ near $t=1$. Then $\mathcal{R}_{k}$ is generated by $1 \in \mathcal{E}_{A}$ and $s \in \mathcal{E}_{A}$ defined by $s(t)=1$ near $t=1$ and $s(t)=0$ near $t=1$, over $k^{*} \mathcal{E}_{2}$. Then $K=(k, s):(\mathbf{R}, A) \rightarrow \mathbf{R}^{3}$ is the versal opening of $k$. In fact $K$ resolves the self-intersection by the over and underpasses. 
Example 2.7 Here we add several additional illustrative examples. Let us consider the following five map-germs: $f:\left(\mathbf{R}^{2}, 0\right) \rightarrow\left(\mathbf{R}^{2}, 0\right), g:\left(\mathbf{R}^{2}, 0\right) \rightarrow$ $\left(\mathbf{R}^{3}, 0\right), h:\left(\mathbf{R}^{2}, 0\right) \rightarrow\left(\mathbf{R}^{3}, 0\right), k:\left(\mathbf{R}^{2}, 0\right) \rightarrow\left(\mathbf{R}^{2}, 0\right), \ell:\left(\mathbf{R}^{2}, 0\right) \rightarrow\left(\mathbf{R}^{2}, 0\right)$ defined by

$$
\begin{gathered}
f(x, t)=\left(x, t^{2}\right), \quad g(x, t)=\left(x, x t, t^{2}\right), \quad h(x, t)=\left(x^{2}, x t, t^{2}\right), \\
k(x, t)=\left(x^{2}, t^{2}\right), \quad \ell(x, t)=\left(x^{2}-t^{2}, x t\right) .
\end{gathered}
$$

Then we have

$$
\mathcal{R}_{k} \subsetneq \mathcal{R}_{h} \subsetneq \mathcal{R}_{g}, \quad \mathcal{R}_{\ell} \subsetneq \mathcal{R}_{h}, \quad \mathcal{R}_{f} \subsetneq \mathcal{R}_{g} .
$$

In fact

$$
\begin{gathered}
\mathcal{J}_{f}=\langle d x, t d t\rangle_{\mathcal{E}_{2}}, \quad \mathcal{J}_{g}=\langle d x, x d t, t d t\rangle_{\mathcal{E}_{2}}, \quad \mathcal{J}_{h}=\langle x d x, x d t+t d x, t d t\rangle_{\mathcal{E}_{2}}, \\
\mathcal{J}_{k}=\langle x d x, t d x\rangle_{\mathcal{E}_{2}}, \quad \mathcal{J}_{\ell}=\langle x d x-t d t, t d x+x d t\rangle_{\mathcal{E}_{2}} .
\end{gathered}
$$

Then we see that $\mathcal{R}_{f}$ is minimally generated by $1, t^{3}$ over $f^{*} \mathcal{E}_{2}, \mathcal{R}_{g}$ is minimally generated by $1, t^{3}$ over $g^{*} \mathcal{E}_{3}$, and $\mathcal{R}_{h}$ is minimally generated by $1, x^{3}, x^{2} t, x t^{2}, t^{3}$ over $h^{*} \mathcal{E}_{3}$. Moreover we have that $\mathcal{R}_{k}$ is minimally generated by $1, x^{3}, t^{3}, x^{3} t^{3}$ over $k^{*} \mathcal{E}_{2}$ and that $\mathcal{R}_{\ell}$ is minimally generated by $1, x^{3}-3 x t^{2}, 3 x^{2} t-t^{3}, x^{2}\left(x^{2}+t^{2}\right)^{2}$ over $\ell^{*} \mathcal{E}_{2}$.

Remark 2.8 (continued with Remark 2.4). Let $\coprod_{s} f_{i}: \coprod_{s}\left(\mathbf{R}^{n}, 0\right) \rightarrow$ $\left(\mathbf{R}^{m}, b\right)$ be a multi-germ of map. Suppose $F_{i}=\left(f_{i} ; h_{i 1}, \ldots, h_{i r_{i}}\right):\left(\mathbf{R}^{n}, 0\right) \rightarrow$ $\left(\mathbf{R}^{m+r_{i}}, b \times 0\right)$ be a versal opening of $f_{i}$. Then, setting $r=\max _{1 \leq i \leq s} r_{i}$ and $h_{i j}=0$ if $j>r_{i}$, then $F=\coprod_{s}\left(f_{i}, h_{i 1}, \ldots, h_{i r_{i}}, \ldots\right): \coprod_{s}\left(\mathbf{R}^{n}, 0\right) \rightarrow$ $\left(\mathbf{R}^{m+r}, b \times 0\right)$ is a versal opening of $\coprod_{s} f_{i}$.

By Proposition 2.5, we have

Corollary 2.9 Let $f:\left(\mathbf{R}^{n}, A\right) \rightarrow\left(\mathbf{R}^{m}, b\right)$ be finite and of corank at most one. Then there exists a versal opening of $f$.

Moreover we have the following:

Corollary 2.10 Let $f:\left(\mathbf{R}^{n}, A\right) \rightarrow\left(\mathbf{R}^{m}, b\right)$ be finite and of corank at most one. Then an opening $F=\left(f, h_{1}, \ldots, h_{r}\right)$ of $f$ is a mini-versal opening of $f$, namely, $1, h_{1}, \ldots, h_{r} \in \mathcal{R}_{f}$ form a minimal system of generators of $\mathcal{R}_{f}$ as $\mathcal{E}_{b}$-module if and only if they form a basis of $\mathbf{R}$-vector space $\mathcal{R}_{f} /\left(f^{*} \mathfrak{m}_{b}\right) \mathcal{R}_{f}$. 
The following is useful for the classification problem of map-germs in a geometric context ([15][16]. See also §3.)

Proposition 2.11 Let $f:\left(\mathbf{R}^{n}, A\right) \rightarrow\left(\mathbf{R}^{m}, b\right), n \leq m$ be a $C^{\infty}$ map-germ. (1) For any versal opening $F:\left(\mathbf{R}^{n}, A\right) \rightarrow\left(\mathbf{R}^{m+r}, F(A)\right)$ of $f$ and for any opening $G:\left(\mathbf{R}^{n}, A\right) \rightarrow\left(\mathbf{R}^{m+s}, G(A)\right)$, there exists an affine bundle map $\Psi:\left(\mathbf{R}^{m+r}, F(A)\right) \rightarrow\left(\mathbf{R}^{m+s}, G(A)\right)$ over $\left(\mathbf{R}^{m}, b\right)$ such that $G=\Psi \circ F$.

(2) For any mini-versal openings $F:\left(\mathbf{R}^{n}, A\right) \rightarrow\left(\mathbf{R}^{m+r}, F(A)\right)$ and $F^{\prime}$ : $\left(\mathbf{R}^{n}, A\right) \rightarrow\left(\mathbf{R}^{m+r}, F^{\prime}(A)\right)$ of $f$, there exists an affine bundle isomorphism $\Phi:\left(\mathbf{R}^{m+r}, F(A)\right) \rightarrow\left(\mathbf{R}^{m+r}, F^{\prime}(A)\right)$ over $\left(\mathbf{R}^{m}, b\right)$ such that $F^{\prime}=\Psi \circ F$. In particular, the diffeomorphism class of mini-versal opening of $f$ is unique.

(3) Any versal openings $F^{\prime \prime}:\left(\mathbf{R}^{n}, A\right) \rightarrow\left(\mathbf{R}^{m+s}, F^{\prime \prime}(A)\right)$ of $f$ is diffeomorphic to $(F, 0)$ for a mini-versal opening $F$ of $f$.

Proof: (1) Let $F=\left(f, h_{1}, \ldots, h_{r}\right)$ and $G=\left(f, k_{1}, \ldots, k_{s}\right)$. Since $k_{j} \in \mathcal{R}_{f}$, there exist $c_{j}{ }^{0}, c_{j}{ }^{1}, \ldots, c_{j}{ }^{r} \in \mathcal{E}_{b}$ such that $k_{j}=c_{j}{ }^{0} \circ f+\left(c_{j}{ }^{1} \circ f\right) h_{1}+\cdots+$ $\left(c_{j}^{r} \circ f\right) h_{r}$. Then it suffices to set $\Psi(y, z)=\left(y,\left(c_{j}{ }^{0}(y)+c_{j}{ }^{1}(y) z_{1}+\cdots+\right.\right.$ $\left.\left.c_{j}^{r}(y) z_{r}\right)_{1 \leq j \leq s}\right)$. (2) By (1) there exists an affine bundle map $\Psi$ with $F^{\prime}=$ $\Psi \circ F$. From the minimality, we have that the matrix $\left(c_{j}{ }^{i}(b)\right)$ is regular. (See Remark 2.10). Therefore $\Psi$ is a diffeomorphism-germ. (3) Let $F=\Psi \circ F^{\prime \prime}$ for some affine bundle map $\Psi$. Then the matrix $\left(c_{j}{ }^{i}(b)\right)$ is of rank $r$. Therefore $F^{\prime \prime}$ is diffeomorphic to $\left(F, k_{1}, \ldots, k_{s-r}\right)$ for some $k_{j} \in \mathcal{R}_{f}$. Write each $k_{j}=$ $K_{j} \circ F$ for some $K_{j} \in \mathcal{E}_{F(a)}$. Then we set $\Xi(y, z, w)=(y, z, w-K \circ F)$. Then $\Xi$ is a local diffeomorphism on $\mathbf{R}^{m+r+(s-r)}$ and $\Xi \circ\left(F, k_{1}, \ldots, k_{s-r}\right)=(F, 0)$.

Two map-germs $F:\left(\mathbf{R}^{n}, A\right) \rightarrow\left(\mathbf{R}^{p}, B\right)$ and $G:\left(\mathbf{R}^{n}, A\right) \rightarrow\left(\mathbf{R}^{q}, C\right)$ is called $\mathcal{L}$-equivalent, or, left-equivalent, if there exists a diffeomorphism-germ $\Psi:\left(\mathbf{R}^{p}, B\right) \rightarrow\left(\mathbf{R}^{q}, C\right)$ such that $G=\Psi \circ F$.

Then, by Proposition 2.11, we have:

Corollary 2.12 Let $f:\left(\mathbf{R}^{n}, A\right) \rightarrow\left(\mathbf{R}^{m}, b\right)$ be a $C^{\infty}$ map-germ $(n \leq m)$. Then a mini-versal opening of $f$ is unique up to $\mathcal{L}$-equivalence. A versal opening of $f$ is $\mathcal{L}$-equivalent to a mini-versal opening composed with an immersion.

Remark 2.13 An opening $F=\left(f, h_{1}, \ldots, h_{s}\right)$ of a map-germ

$$
f=\left(f_{1}, \ldots, f_{m}\right):\left(\mathbf{R}^{n}, A\right) \rightarrow\left(\mathbf{R}^{m}, b\right)
$$


is called $\mathcal{L}$-minimal if $f_{1}, \ldots, f_{m}, h_{1}, \ldots, h_{r}$ minimally generate the $C^{\infty}$-ring $\mathcal{R}_{f}$ over $f^{*} \mathcal{E}_{\mathbf{R}^{m}, b}$. Then we have a similar uniqueness result for $\mathcal{L}$-equivalence of $\mathcal{L}$-minimal openings.

In Example 2.7, we have seen that $1, x^{3}, t^{3}, x^{3} t^{3}$ minimally generate $\mathcal{R}_{k}$ as $k^{*} \mathcal{E}_{2}$-module. However $1, x^{3}, t^{3}$ already minimally generate $\mathcal{R}_{k}$ as $k^{*} \mathcal{E}_{2^{-}}$ $C^{\infty}$-ring. Therefore $K=\left(x^{2}, y^{2}, x^{3}, t^{3}, x^{3} t^{3}\right)$ is a mini-versal opening of $k=\left(x^{2}, y^{2}\right):\left(\mathbf{R}^{2}, 0\right) \rightarrow\left(\mathbf{R}^{2}, 0\right)$ and $K^{\prime}=\left(x^{2}, y^{2}, x^{3}, t^{3}\right)$ is a $\mathcal{L}$-minimal opening of $k$.

Lastly we show injectivity of versal openings:

Proposition 2.14 Let $f:\left(\mathbf{R}^{n}, A\right) \rightarrow\left(\mathbf{R}^{m}, b\right)$ be a finite map-germ. Suppose $F:\left(\mathbf{R}^{n}, A\right) \rightarrow\left(\mathbf{R}^{m+r}, F(A)\right)$ is a versal opening of $f$. Then $F$ has an injective representative.

Proof: Let $A=\left\{a_{1}, \ldots a_{s}\right\}$. If each restriction $\left.F\right|_{\left(\mathbf{R}^{n}, a_{i}\right)}$ for $i=1,2, \ldots, s$ is injective, then $F$ is injective. In fact, for each $i$ and for each $v \in \mathbf{R}^{m}$, we can take a map-germ $F^{i, v}:\left(\mathbf{R}^{n}, A\right) \rightarrow\left(\mathbf{R}^{m}, F_{i}(A)\right)$ which coincides with $F$ near $a_{j},(j \neq i)$ and $F+v$ near $a_{i}$. Since any $F^{i, v}$ is an opening of $f$, and $F$ is a versal opening, different branches of $F$ can not intersect each other. (See Proposition 2.11 (1)).

Now suppose that $f$ is a mono-germ $f:\left(\mathbf{R}^{n}, a\right) \rightarrow\left(\mathbf{R}^{m}, b\right)$. Assume the versal opening map-germ $F:\left(\mathbf{R}^{n}, a\right) \rightarrow\left(\mathbf{R}^{m}, F(a)\right)$ of $f$ has no injective representative. Then there must be a sequence of points $b_{i}$ in $\mathbf{R}^{m}$ which tends to $F(a)$ when $i \rightarrow \infty$, and $a_{i}^{1}, a_{i}^{2}$ in $\mathbf{R}^{n}$ which tend to $a$ respectively when $i \rightarrow \infty$, such that $F\left(a_{i}^{1}\right)=F\left(a_{i}^{2}\right)=b_{i}$. We may suppose $b_{i} / b_{j}$ if $i \neq j$. Take a $C^{\infty}$ function $h$ on $\mathbf{R}^{m}$ such that the support is a disjoint union of small balls centred at $b_{i}, i=1,2, \ldots$. Note that such function must satisfy $h(b)=0$. Take the $C^{\infty}$ function $k=F^{*} h$ on $\mathbf{R}^{n}$. Since $F$ is finite, the support of $k$ is a disjoint union of closed neighbourhoods of $a_{i}^{1}$ and those of $a_{i}^{2}$. Take the function $k^{\prime}$ which coincides with $k$ except on the closed neighbourhoods of $a_{i}^{2}$, and is identically zero there. Then we see that $k^{\prime}$ is $C^{\infty}, k^{\prime}$ belongs to $\mathcal{R}_{F}=\mathcal{R}_{f}$ and $k^{\prime}\left(a_{i}^{1}\right) \neq k^{\prime}\left(a_{i}^{2}\right)$ for any $i=1,2, \ldots$ Consider the opening $\left(f, k^{\prime}\right):\left(\mathbf{R}^{n}, a\right) \rightarrow\left(\mathbf{R}^{m+1},(b, 0)\right.$ of $f$. This leads a contradiction, since $F$ is a versal opening of $f$. (See Proposition 2.11 (1), or, the definition of versal openings Definition 1.2).

Related to the above Proposition 2.14, T. Gaffney suggested a relation of the notion of openings and that of weak normalisations [6], which is left to be our open problem. 


\section{Related geometric problems}

Let $n \leq m$. A $C^{\infty}$ mapping $f: N^{n} \rightarrow M^{m}$ is called frontal if (I) the locus of regular points

$$
\operatorname{Reg}(f)=\{x \in N \mid f:(N, x) \rightarrow(M, f(x)) \text { is immersive }\}
$$

of $f$ is dense in $N$.

(II) There exists a $C^{\infty}$ mapping $\widetilde{f}: N \rightarrow \operatorname{Gr}(n, T M)$ satisfying $\widetilde{f}(x)=$ $f_{*}\left(T_{x} N\right)$ for any $x \in \operatorname{Reg}(f)$.

Here $\operatorname{Gr}(n, T M)=\bigcup_{y \in M} \operatorname{Gr}\left(n, T_{y} M\right)$ is the Grassmannian bundle consisting of $n$-dimensional linear subspaces in fibres of $T M$.

The mapping $\widetilde{f}$ is uniquely determined if it exists, which we call the Grassmann lifting of $f$.

Recall that $\operatorname{Gr}(n, T M)$ is endowed with the canonical differential system $D \subset T \operatorname{Gr}(n, T M)$ defined as follows: We denote by $\pi: \operatorname{Gr}(n, T M) \rightarrow M$ the projection defined by $\pi(y, L)=y$, for $(y, L) \in \operatorname{Gr}(n, T M)$. For each $(y, L) \in$ $\operatorname{Gr}(n, T M)$ and for each tangent vector $v \in T_{(y, L)} \operatorname{Gr}(n, T M), v \in D_{(y, L)}$ if and only if $\pi_{*}(v) \in L\left(\subset T_{y} M\right)$, where $\pi_{*}: T_{(y, L)} \operatorname{Gr}(n, T M) \rightarrow T_{y} M$ the differential of $\pi$ at $(y, L)$.

The Grassmann lifting $\tilde{f}$ of a frontal mapping $f: N \rightarrow M$ is an integral mapping to the canonical differential system $D$ on $\operatorname{Gr}(n, T M)$ and satisfies that $\pi \circ \tilde{f}=f$.

If $f$ is an immersion, then $f$ is frontal. For example, the tangent mapping, a parametrization of tangent surface to a curve of finite type is a non-immersive frontal [15].

If $f$ is frontal and $\widetilde{f}$ is an immersion, then $f$ is called a front.

Let $f:(N, A) \rightarrow(M, b)$ be a multi-germ of frontal mapping, $A$ being a finite set of $N$. Then, since $\widetilde{f}(a)$, for each $a \in A$, is an $n$-dimensional vector subspace of $T_{b} M$, there exists of a system of local coordinates of $(M, b)$ $y_{1}, \ldots, y_{n}, z_{1}, \ldots, z_{k},(k=m-n)$, such that, for $g=\left(y_{1} \circ f, \ldots, y_{n} \circ f\right)$, each component $z_{j} \circ f,(1 \leq j \leq k)$ belongs to the ramification module $\mathcal{R}_{g}$ and therefore $f$ is regarded as an opening of $g$. Then the regular points locus $\operatorname{Reg}(g)=\operatorname{Reg}(f)$ is dense in $(N, A)$. Conversely, if the regular points locus $\operatorname{Reg}(g)$ of a given map-germ $g=\left(g_{1}, \ldots, g_{n}\right):(N, A) \rightarrow \mathbf{R}^{n}$ is dense in $(N, A)$, then any opening of $g$ is frontal. In particular, an opening of a frontal mapping is frontal.

Let $M$ be a $(2 m+1)$-dimensional contact manifold with the contact structure $D$. Recall that, by Darboux's theorem, $D$ is locally defined by $d y-\sum_{i=1}^{n} p_{i} d x_{i}=0$ for a system of local coordinates $x_{1}, \ldots, x_{n}, p_{1}, \ldots, p_{n}, y$ 
of $M$. A mapping $f: N^{n} \rightarrow M$ is called a contact integral mapping if $f_{*} T N \subset D$. Suppose $f$ is a contact integral mapping and $A \subset N$ is a finite set such that $f(A)$ is one point which belongs to a coordinate neighbourhood as above. Then the $y$-components belong to the ramification module $\mathcal{R}_{g}$, for $g=\left(x_{1}, \ldots, x_{n}\right) \circ f$. Therefore $(x, y) \circ f=(g, y \circ f)$ is an opening of $g$. Moreover if $\operatorname{Reg}(g)$ is dense, then $(x, y) \circ f$ is a frontal mapping.

The notion of openings has a close relation also to the singularity theory on "isotropic" map-germs in a symplectic space.

Let $\mathbf{R}^{2 m}$ be the standard symplectic space endowed with the symplectic form $\omega=\sum_{i=1}^{m} d p_{i} \wedge d x_{i}$ for the coordinates $x_{1}, \ldots, x_{m}, p_{1}, \ldots, p_{m}$. Let $\pi: \mathbf{R}^{2 m} \rightarrow \mathbf{R}^{m}$ be the Lagrangian projection defined by $\pi(x, p)=x$. A map-germ $f:\left(\mathbf{R}^{n}, A\right) \rightarrow \mathbf{R}^{2 m}$ is called isotropic if $f^{*} \omega=0$. If we consider the Liouville form $\theta=\sum_{i=1}^{m} p_{i} d x_{i}$, then $d \theta=\omega$, so $d\left(f^{*} \theta\right)=0$ for an isotropic $f$. Then there exists a function-germ $e:\left(\mathbf{R}^{m}, A\right) \rightarrow \mathbf{R}$ such that $d e=f^{*} \theta=\sum_{i=1}^{m}\left(p_{i} \circ f\right) d\left(x_{i} \circ f\right)$. Note that $e$ is determined up to a constant addition. Then $e \in \mathcal{R}_{g}, g=\left(x_{1} \circ f, \ldots, x_{m} \circ f\right):\left(\mathbf{R}^{n}, A\right) \rightarrow \mathbf{R}^{m}$. Therefore $(g, e)$ is an opening of $g$. If the locus of immersion $\operatorname{Reg}(g)$ of $g$ is dense in $\mathbf{R}^{n}$ near $A$, then we call $(g, e):\left(\mathbf{R}^{n}, A\right) \rightarrow \mathbf{R}^{m+1}$ the frontal of $f$. Its Grassmann lifting (Legendre lifting) is given by $(f, e)$. The mapping $(f, e)$ is called a Legendrisation of $f$. For example the frontal of a open Whitney-Morin umbrella is the folded umbrella.

\section{Unfoldings and openings}

We recall the notion of unfolding of map-germs ([19]).

Let $f:\left(\mathbf{R}^{n}, A\right) \rightarrow\left(\mathbf{R}^{m}, b\right)$ be a map-germ. An unfolding of $f$ is a mapgerm $F:\left(\mathbf{R}^{n+\ell}, A \times 0\right) \rightarrow\left(\mathbf{R}^{m+\ell},(b, 0)\right)$ of form $F(x, u)=\left(F_{1}(x, u), u\right)$ and $F_{1}(x, 0)=f(x)$, for $(x, u) \in\left(\mathbf{R}^{n+\ell}, A \times 0\right)$.

For another unfolding $G:\left(\mathbf{R}^{n+\ell}, A \times 0\right) \rightarrow\left(\mathbf{R}^{m+\ell},(b, 0)\right), F$ and $G$ are called isomorphic if there exist an unfolding $\Sigma:\left(\mathbf{R}^{n+\ell}, A \times 0\right) \rightarrow\left(\mathbf{R}^{n+\ell}, A \times\right.$ $0)$ of the identity map on $\left(\mathbf{R}^{n}, A\right)$ and an unfolding $T:\left(\mathbf{R}^{m+\ell},(b, 0)\right) \rightarrow$ $\left(\mathbf{R}^{m+\ell},(b, 0)\right)$ of the identity map on $\left(\mathbf{R}^{m}, b\right)$ such that $G \circ \Sigma=T \circ F$.

Proposition 4.1 (Unfoldings and openings) Let $f:\left(\mathbf{R}^{n}, A\right) \rightarrow\left(\mathbf{R}^{m}, b\right)$ be $a C^{\infty}$ map-germ and $F:\left(\mathbf{R}^{n+\ell}, A \times 0\right) \rightarrow\left(\mathbf{R}^{m+\ell},(b, 0)\right)$ be an unfolding of $f$. Let $i:\left(\mathbf{R}^{n}, A\right) \rightarrow\left(\mathbf{R}^{n+\ell}, A \times 0\right)$ be the inclusion, $i(x)=(x, 0)$. Then we have:

(1) $i^{*} \mathcal{R}_{F} \subset \mathcal{R}_{f}$.

(2) If $f$ is of corank $\leq 1$ with $n \leq m$, then $i^{*} \mathcal{R}_{F}=\mathcal{R}_{f}$. If $1, H_{1}, \ldots, H_{r}$ generate $\mathcal{R}_{F}$ via $F^{*}$, then $1, i^{*} H_{1}, \ldots, i^{*} H_{r}$ generate $\mathcal{R}_{f}$ via $f^{*}$. 
Proof: For the mono-germ case the assertions are proved in Proposition 1.6 of [12], Lemma 2.4 of [13]. Here we present the proof for the general case: (1) is clear. (2) Let $H \in \mathcal{R}_{F}$. Then $d H \in \mathcal{J}_{F}$. Hence $d\left(i^{*} H\right)=i^{*}(d H) \in$ $i^{*} \mathcal{J}_{F} \subset \mathcal{J}_{f}$. Therefore $i^{*} H \in \mathcal{R}_{f}$. Let $f$ be of corank at most one. Suppose $h \in \mathcal{R}_{f}$. Then $d h=\sum_{j=1}^{m} a_{j} d f_{j}$ for some $a_{j} \in \mathcal{E}_{a}$. There exist $A_{j}, B_{k} \in \mathcal{E}_{(a, 0)}$ such that $i^{*} A_{j}=a_{j}$ and the 1 -form $\sum_{j=1}^{m} A_{j} d\left(F_{1}\right)_{j}+\sum_{k=1}^{\ell} B_{k} d \lambda_{k}$ is closed (cf. Lemma 2.5 of [14]). Then there exists an $H \in \mathcal{E}_{(a, 0)}$ such that $d H=$ $\sum_{j=1}^{m} A_{j} d\left(F_{1}\right)_{j}+\sum_{k=1}^{\ell} B_{k} d \lambda_{k} \in \mathcal{J}_{F}$ and $d\left(i^{*} H\right)=i^{*}(d H)=d h$. Then there exists $c \in \mathbf{R}$ such that $h=i^{*} H+c=i^{*}(H+c)$, and $H+c \in \mathcal{R}_{F}$. Therefore $h \in i^{*} \mathcal{R}_{F}$. Since $i^{*}$ is a homomorphism over $j^{*}: \mathcal{E}_{(b, 0)} \rightarrow \mathcal{E}_{b}$, where $j:\left(\mathbf{R}^{m}, 0\right) \rightarrow\left(\mathbf{R}^{m+\ell}, 0\right)$ is the inclusion $j(y)=(y, 0)$, we have the consequence.

An unfolding $F:\left(\mathbf{R}^{n+\ell}, A \times 0\right) \rightarrow\left(\mathbf{R}^{m+\ell},(b, 0)\right)$ of a map-germ $f:$ $\left(\mathbf{R}^{n}, A\right) \rightarrow\left(\mathbf{R}^{m}, b\right)$ is called extendable if $i^{*} \mathcal{R}_{F}=\mathcal{R}_{f}$ for the inclusion $i:\left(\mathbf{R}^{n}, A\right) \rightarrow\left(\mathbf{R}^{n+\ell}, A \times 0\right)$. By Proposition 4.1, we have:

Corollary 4.2 If corank of $f$ is at most one, then any unfolding of $f$ is extendable.

In $\S 7$, we will see that there exist non-extendable unfoldings for mapgerms of corank $\geq 2$. Therefore the opening constructions do not behave well under unfoldings in general.

\section{Openings of stable maps of corank one}

We will give the explicit versal opening in the case of corank one. As is seen in Remark 2.8, it is sufficient to treat the case of mono-germs, namely, germs $f:\left(\mathbf{R}^{n}, 0\right) \rightarrow\left(\mathbf{R}^{m}, 0\right)$ of corank one. Moreover, by Corollary 4.2, it is sufficient to treat the case that $f$ is stable, namely, $f$ is a Morin map.

Let $k \geq 0, m \geq 0$. To present the normal forms of Morn maps, consider variables $t, \lambda=\left(\lambda_{1}, \ldots, \lambda_{k-1}\right), \mu=\left(\mu_{i j}\right)_{1 \leq i \leq m, 1 \leq j \leq k}$ and polynomials

$$
F(t, \lambda)=t^{k+1}+\sum_{i=1}^{k-1} \lambda_{j} t^{j}, \quad G_{i}(t, \mu)=\sum_{j=1}^{k} \mu_{i j} t^{j},(1 \leq i \leq m) .
$$

Let $f:\left(\mathbf{R}^{k+k m}, 0\right) \rightarrow\left(\mathbf{R}^{m+k+k m}, 0\right)$ be a Morin map defined by

$$
f(t, \lambda, \mu):=(F(t, \lambda), G(t, \mu), \lambda, \mu),
$$


for the above polynomials $F$ and $G$.

For $\ell \geq 0$, we denote by $F_{(\ell)}, G_{i(\ell)}$ the polynomials

$$
F_{(\ell)}(t, \lambda)=\int_{0}^{t} s^{\ell} F(s, \lambda) d s, \quad G_{i(\ell)}(t, \mu)=\int_{0}^{t} s^{\ell} G_{i}(s, \mu) d s .
$$

Then we have:

Proposition 5.1 (Theorem 3 of [11]) The ramification module $\mathcal{R}_{f}$ of the Morin map $f$ is minimally generated over $f^{*} \mathcal{E}_{m+k+k m}$ by the $1+k+(k-1) m$ elements

$$
1, F_{(1)}, \ldots, F_{(k)}, G_{1(1)}, \ldots, G_{1(k-1)}, \ldots, G_{m(1)}, \ldots, G_{m(k-1)} .
$$

The map-germ $\mathbf{F}:\left(\mathbf{R}^{k+m k}, 0\right) \rightarrow\left(\mathbf{R}^{m+k+k m} \times \mathbf{R}^{k+(k-1) m}, 0\right)=\left(\mathbf{R}^{2(k+k m)}, 0\right)$ defined by

$$
\mathbf{F}=\left(f, F_{(1)}, \ldots, F_{(k)}, G_{1(1)}, \ldots, G_{1(k-1)}, \ldots, G_{m(1)}, \ldots, G_{m(k-1)}\right)
$$

is a mini-versal opening of $f$.

Proof: The first half is proved in [11]. The second half follows from the definition.

Remark 5.2 It is shown in [11] moreover that $\mathbf{F}$ is an isotropic map for a symplectic structure on $\mathbf{R}^{2(k+k m)}$.

In particular we have:

Lemma 5.3 Let $\ell$ be a positive integer and $F=\left(F_{1}(t, u), u\right):\left(\mathbf{R}^{n}, 0\right) \rightarrow$ $\left(\mathbf{R}^{n}, 0\right)$ an unfolding of $f:(\mathbf{R}, 0) \rightarrow(\mathbf{R}, 0), f(t)=F_{1}(t, 0)=t^{\ell}$. Suppose $H_{1}, \ldots, H_{r} \in \mathcal{R}_{F} \cap \mathfrak{m}_{n}$. Then $1, H_{1}, \ldots, H_{r}$ generate $\mathcal{R}_{F}$ via $F^{*}$ if and only $i^{*} H_{1}, \ldots, i^{*} H_{r}$ generate $\mathfrak{m}_{1}^{\ell+1} / \mathfrak{m}_{1}^{2 \ell}$. In particular $F_{1(1)}, \ldots, F_{1(\ell-1)}$ form a system of generators of $\mathcal{R}_{F}$ via $F^{*}$ over $\mathcal{E}_{n}$.

Proof: It is easy to show that $\mathcal{R}_{f}=\mathbf{R}+\mathfrak{m}_{1}^{\ell}$. By Proposition 2.5 (2), $1, H_{1}, \ldots, H_{r}$ generate $\mathcal{R}_{F}$ as $\mathcal{E}_{n}$-module via $F^{*}$ if and only if they generate $\mathcal{R}_{F} / F^{*}\left(\mathfrak{m}_{n}\right) \mathcal{R}_{F}$ over $\mathbf{R}$. Since

$$
\mathcal{R}_{F} / F^{*}\left(\mathfrak{m}_{n}\right) \mathcal{R}_{F} \cong\left(\mathbf{R}+\mathfrak{m}_{1}^{\ell}\right) /\left(f^{*} \mathfrak{m}_{1}\right)\left(\mathbf{R}+\mathfrak{m}_{1}^{\ell}\right) \cong \mathfrak{m}_{1}^{\ell+1} / \mathfrak{m}_{1}^{2 \ell}
$$

we have the consequence. 


\section{Versal openings of analytic map-germs}

In this section we discuss the case $f$ is analytic.

First we recall the complex analytic case briefly from [12].

Let $(X, A)$ be a germ of complex analytic space at a finite set $A$ with the structure sheaf $\mathcal{O}_{X, A}$, and $f=\left(f_{1}, \ldots, f_{m}\right):(X, A) \rightarrow\left(\mathbf{C}^{m}, b\right)$ a finite analytic map-germ. In the graded differential $\mathcal{O}_{X, A}$-algebra (de Rham algebra) $\Omega_{X, A}$ on $(X, A)$, consider the graded differential ideal $\mathcal{I}_{f}$ generated by $d f_{1}, \ldots, d f_{m}$. Then the differential $d$ on $\Omega_{X, A}$ induces the $f^{*} \mathcal{O}_{\mathbf{C}^{m-}}$ homomorphism $d: \Omega_{X, A} / \mathcal{I}_{f} \rightarrow \Omega_{X, A} / \mathcal{I}_{f}$ and then $\mathcal{O}_{\mathbf{C}^{m}, b}$-homomorphism $d: f_{*}\left(\Omega_{X, A} / \mathcal{I}_{f}\right) \rightarrow f_{*}\left(\Omega_{X, A} / \mathcal{I}_{f}\right)$. Then we consider the $i$-th cohomology $\mathcal{H}^{i}\left(f_{*}\left(\Omega_{X, A} / \mathcal{I}_{f}\right) ; d\right)$ for the complex $\left(f_{*}\left(\Omega_{X, A} / \mathcal{I}_{f}\right), d\right)$. We observe

Proposition 6.1 (Proposition 1.1 of $[12]) \mathcal{H}^{i}\left(f_{*}\left(\Omega_{X, A} / \mathcal{I}_{f}\right) ; d\right)$ is a coherent $\mathcal{O}_{\mathbf{C}^{m}, b}$-module $(i=0,1,2, \ldots)$.

We remark that the stalk of $\mathcal{H}^{0}\left(f_{*}\left(\Omega_{X, A} / \mathcal{I}_{f}\right) ; d\right)$ at $A$ is the complex analytic counterpart of $\mathcal{R}_{f}$ in the real $C^{\infty}$ case. We write it $\mathcal{R}_{f}^{\text {hol }}$ to distinguish with the real $C^{\infty}$ case. By Proposition 6.1, in particular, that $\mathcal{R}_{f}^{\text {hol }}$ is a finite $\mathcal{O}_{\mathbf{C}^{m}}$-module.

Now let $f:\left(\mathbf{R}^{n}, A\right) \rightarrow\left(\mathbf{R}^{m}, b\right)$ be a finite real analytic map-germ ( $(2)$. We denote by $\mathcal{O}_{\mathbf{R}^{n}, A}$ (resp. $\mathcal{O}_{\mathbf{R}^{m}, b}$ ) the germ of sheaf of analytic functions on $\left(\mathbf{R}^{n}, A\right)$ (resp. $\left.\left(\mathbf{R}^{m}, b\right)\right)$. Then, besides with $\mathcal{R}_{f}$, we consider the sheaf

$$
\mathcal{R}_{f}^{\omega}:=\left\{h \in \mathcal{O}_{\mathbf{R}^{n}, A} \mid d h \in\left\langle d f_{1}, \ldots, d f_{m}\right\rangle_{\mathcal{O}_{\mathbf{R}^{n}, A}}\right\}
$$

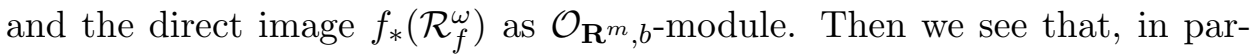
ticular, $f_{*}\left(\mathcal{R}_{f}^{\omega}\right)$ is a finite $\mathcal{O}_{\mathbf{R}^{m}, b}$-module by Proposition 6.1 in the case $X=\left(\mathbf{C}^{n}, A\right)$. Thus we have that $f_{*}\left(\mathcal{R}_{f}^{\omega}\right)$ is generated over $\mathcal{O}_{\mathbf{R}^{m}, b}$ by some $1, h_{1}, \ldots, h_{r} \in \mathcal{R}_{f}^{\omega}$. Moreover we see that $F=\left(f, h_{1}, \ldots, h_{r}\right):\left(\mathbf{R}^{n}, A\right) \rightarrow$ $\left(\mathbf{R}^{m+r}, b \times h(A)\right)$ is injective (See [12]).

Then we show the following:

Proposition 6.2 Let $f:\left(\mathbf{R}^{n}, 0\right) \rightarrow\left(\mathbf{R}^{m}, 0\right)$ be a finite analytic mono map-germ. Suppose $1, h_{1}, \ldots, h_{r}$ generate $\mathcal{R}_{f}^{\omega}$ over $\mathcal{O}_{\mathbf{R}^{m}, 0}$ via $f^{*}$. Then $1, h_{1}, \ldots, h_{r}$ generate $\mathcal{R}_{f}$ over $\mathcal{E}_{\mathbf{R}^{m}, 0}$ via $f^{*}$.

Proof: First we may suppose $h_{i}(0)=0,(1 \leq i \leq r)$. Then we remark that the opening

$$
F=\left(f_{1}, \ldots, f_{m}, h_{1}, \ldots, h_{r}\right):\left(\mathbf{R}^{n}, 0\right) \rightarrow\left(\mathbf{R}^{m+r}, 0\right)
$$


of $f$ is injective.

Let $\mathcal{F}_{p}$ stand for the $\mathbf{R}$-algebra of formal functions on $\left(\mathbf{R}^{n}, p\right)$, and set $\widetilde{\mathcal{F}}_{n}=\prod_{p \in\left(\mathbf{R}^{n}, 0\right)} \mathcal{F}_{p}$. Then $\widetilde{\mathcal{F}}$ is faithfully flat over $\mathcal{O}_{n}$ (Cor. 4.13 of [18]). Define the formal counterpart

$$
\widetilde{\mathcal{R}}_{f}=\left\{\left(\widehat{h}_{p}\right)_{p \in\left(\mathbf{R}^{n}, 0\right)} \in \widetilde{\mathcal{F}}_{n} \mid d h_{p} \in\left\langle d \widehat{f}_{1, p}, \ldots, d \widehat{f}_{n, p}\right\rangle_{\mathcal{F}_{p}}, p \in\left(\mathbf{R}^{n}, 0\right)\right\}
$$

of $\mathcal{R}_{f}$. Then $1, h_{1}, \ldots, h_{r}$ generate $\widetilde{\mathcal{R}}_{f}$ over $\widetilde{\mathcal{F}}_{m}$. Then, since $F$ is injective, we have $\mathcal{R}_{f} \subseteq F^{*} \mathcal{E}_{m+r}$ by Gleaser's type theorem (see [2][3]). Since $F$ is an opening, we have $\mathcal{R}_{f}=F^{*} \mathcal{E}_{m+r}$

Let $\pi:\left(\mathbf{R}^{m+r}, 0\right) \rightarrow\left(\mathbf{R}^{m}, 0\right)$ be the projection. Then $\pi^{*}: \mathcal{E}_{m} \rightarrow \mathcal{E}_{m+r}$ is the inclusion. Then regard $F^{*} \mathcal{E}_{m+r}$ as an $\mathcal{E}_{m+r}$-module via $F^{*}$. By the preparation theorem, $1, h_{1}, \ldots, h_{r}$ generate $F^{*} \mathcal{E}_{m+r}=\mathcal{R}_{f}$ as $\mathcal{E}_{m}$-module via $F^{*} \circ \pi^{*}=f^{*}$ if $1, h_{1}, \ldots, h_{r}$ generate $F^{*} \mathcal{E}_{m+r} /\left(f^{*} \mathfrak{m}_{m}\right) F^{*} \mathcal{E}_{m+r}$ over $\mathbf{R}$. We will show

$$
\mathfrak{m}_{n}^{\infty} \cap F^{*} \mathcal{E}_{m+r} \subseteq\left(f^{*} \mathfrak{m}_{m}\right) F^{*} \mathcal{E}_{m+r}
$$

Set $h=\sum_{i=1}^{m} f_{i}^{2}:\left(\mathbf{R}^{n}, 0\right) \rightarrow(\mathbf{R}, 0)$. Since $f$ is finite, $h^{-1}(0)=\{0\}$, and moreover, the norms of $1 / h$ and its partial derivatives up to say $\ell$ are estimated from above $1 /\|x\|^{\alpha}$ for some $\alpha=\alpha(\ell)>0$.

Now let $k \in \mathfrak{m}_{n}^{\infty} \cap F^{*} \mathcal{E}_{m+r}$. Then $k / h$ is regarded as a $C^{\infty}$ function on $\left(\mathbf{R}^{n}, 0\right)$ and an element of $\mathfrak{m}_{n}^{\infty}$ (see [18]). Moreover $k / h \in F^{*} \mathcal{E}_{m+r}$. Then

$$
k=\left(\sum_{i=1}^{m} f_{i}^{2}\right)(k / h)=\sum_{i=1}^{m} f_{i}\left(f_{i} k / h\right) \in\left(f^{*} \mathfrak{m}_{m}\right) F^{*} \mathcal{E}_{m+r} .
$$

Now let $h \in \mathcal{R}_{f}$ again. Then

$$
\begin{aligned}
h & \equiv a_{0} \circ f+a_{1} \circ f \cdot h_{1}+\cdots+a_{r} \circ f \cdot h_{r} \\
& \equiv a_{0}(0)+a_{1}(0) h_{1}+\cdots+a_{r}(0) h_{r},
\end{aligned}
$$

modulo $\left(f^{*} \mathfrak{m}_{m}\right) F^{*} \mathcal{E}_{m+r}+\mathfrak{m}_{n}^{\infty} \cap F^{*} \mathcal{E}_{m+r} \subseteq\left(f^{*} \mathfrak{m}_{m}\right) F^{*} \mathcal{E}_{m+r}$.

Thus we see $1, h_{1}, \ldots, h_{r}$ generate $F^{*} \mathcal{E}_{m+r} /\left(f^{*} \mathfrak{m}_{m}\right) F^{*} \mathcal{E}_{m+r}$ over $\mathbf{R}$, and they generate $\mathcal{R}_{f}$ over $\mathcal{E}_{\mathbf{R}^{m}, 0}$ via $f^{*}$.

\section{$7 \quad$ The cases of corank $\geq 2$}

If $\operatorname{corank}(f) \geq 2$, then the restriction of a versal opening of an unfolding of $f$ is not necessarily a versal opening of $f$. That phenomenon was observed already in [14]. We utilise Proposition 6.2 if necessary to treat the following examples. 
Example 7.1 (cf. Example 2.7.) Let $f:\left(\mathbf{R}^{2}, 0\right) \rightarrow\left(\mathbf{R}^{2}, 0\right), h(x, y)=$ $\left(\frac{1}{2} x^{2}, \frac{1}{2} y^{2}\right)=(z, w)$. Then $\mathcal{R}_{f}$ is minimally generated by $1, x^{3}, y^{3}, x^{3} y^{3}$ over $f^{*} \mathcal{E}_{\mathbf{R}^{2}, 0}$. Therefore if we set $F:\left(\mathbf{R}^{2}, 0\right) \rightarrow\left(\mathbf{R}^{5}, 0\right)$ by

$$
F(x, y)=\left(\frac{1}{2} x^{2}, \frac{1}{2} y^{2}, x^{3}, y^{3}, x^{3} y^{3}\right),
$$

then $F$ is the mini-versal opening of $f$.

Here we give a concrete method to find the minimal generators as above.

Let $h \in \mathcal{E}_{\mathbf{R}^{2}, 0}=\mathcal{E}_{2}$. Then by the preparation theorem we have

$$
h \equiv(a \circ f) x+(b \circ f) y+(c \circ f) x y,\left(\bmod . f^{*} \mathcal{E}_{2}\right) .
$$

The condition that $h \in \mathcal{R}_{f}$ is equivalent to that $d h$ belongs to Jacobi module $\mathcal{J}_{f}$. We calculate

$$
d h \equiv(a \circ f) d x+(b \circ f) d y+(c \circ f)(y d x+x d y),\left(\bmod . \mathcal{J}_{f}\right),
$$

and set

$$
(a \circ f) d x+(b \circ f) d y+(c \circ f)(y d x+x d y)=A x d x+B y d y,
$$

for some function $A, B \in \mathcal{E}_{2}$. Again by the preparation theorem, we put

$$
\begin{aligned}
& A=\left(a_{1} \circ f\right)+\left(a_{2} \circ f\right) x+\left(a_{3} \circ f\right) y+\left(a_{4} \circ f\right) x y \\
& B=\left(b_{1} \circ f\right)+\left(b_{2} \circ f\right) x+\left(b_{3} \circ f\right) y+\left(b_{4} \circ f\right) x y .
\end{aligned}
$$

Then

$$
\begin{aligned}
A x & =\left(a_{1} \circ f\right) x+\left(a_{2} \circ f\right) x^{2}+\left(a_{3} \circ f\right) x y+\left(a_{4} \circ f\right) x^{2} y \\
& =2\left(z a_{2}\right)+a_{1} x+2\left(z a_{4}\right) y+a_{3} x y, \\
B y & =\left(b_{1} \circ f\right) y+\left(b_{2} \circ f\right) x y+\left(b_{3} \circ f\right) y^{2}+\left(b_{4} \circ f\right) x y^{2} \\
& =2\left(w b_{3}\right)+2\left(w b_{4}\right)+b_{1} y+b_{2} x y .
\end{aligned}
$$

omitting "of", where $z=\frac{1}{2} x^{2}$ and $w=\frac{1}{2} y^{2}$. Then we have

$$
\begin{aligned}
& a+c y=2 z a_{2}+a_{1} x+\left(2 z a_{4}\right) y+a_{3} x y, \\
& b+c x=2 w b_{3}+2\left(w b_{4}\right)+b_{1} y+b_{2} x y .
\end{aligned}
$$

and therefore

$$
\begin{aligned}
& \left(a-2 z a_{2}\right)+\left(-a_{1}\right) x+\left(c-2 z a_{4}\right) y+\left(-a_{3}\right) x y=0, \\
& \left(b-2 w b_{3}\right)+\left(c-2 w b_{4}\right) x+\left(-b_{1}\right) y+\left(-b_{2}\right) x y=0 .
\end{aligned}
$$


Since $\mathcal{E}_{2}$ is free over $f^{*} \mathcal{E}$ in this example, we have

$$
a=2 z a_{2}, a_{1}=0, c=2 z a_{4}, a_{3}=0, b=2 w b_{3}, c=2 w b_{4}, b_{1}=0, b_{2}=0 .
$$

Then we have $\left(a_{4}, b_{4}\right)=k(w, z)$ for a function $k \in \mathcal{E}_{2}$ and

$$
h \equiv\left(2 z a_{2} \circ f\right) x+\left(2 w b_{3} \circ f\right) y+(2 z w k \circ f) x y,\left(\bmod . f^{*} \mathcal{E}_{2}\right) .
$$

Thus we find a minimal system of generators $1,2 z x, 2 w y, 2 z w x y$, namely $1, x^{3}, y^{3}, x^{3} y^{3}$ of $\mathcal{R}_{f}$ over $f^{*} \mathcal{E}_{2}$.

In each case of the following three examples, we have the mini-versal openings using Proposition 6.2.

Example 7.2 Let $g:\left(\mathbf{R}^{3}, 0\right) \rightarrow\left(\mathbf{R}^{3}, 0\right)$ be a map-germ defined by

$$
g(x, y, u)=(z, w, u)=\left(\frac{1}{2} x^{2}+u y, \frac{1}{2} y^{2}+u x, u\right),
$$

which is an unfolding of $f$ in Example 7.1. Then $\mathcal{R}_{g}$ is minimally generated over $g^{*} \mathcal{E}_{\mathbf{R}^{3}, 0}$ by 1 and

$$
\begin{aligned}
\psi_{3} & =x^{3}+y^{3}+3 x y u \\
\psi_{5}^{5,0} & =x^{5}+5 x^{3} y u-12 x^{2} u^{3}+9 y u^{4} \\
\psi_{5}^{0,5} & =y^{5}+5 x y^{3} u-12 y^{2} u^{3}+9 x u^{4} \\
\psi_{6}^{3,3} & =x^{3} y^{3}-12 x^{2} y^{2} u^{2}-11 x^{3} u^{3}-11 y^{3} u^{3}-12 x y u^{4} .
\end{aligned}
$$

Therefore $i^{*} \mathcal{R}_{g} \subsetneq \mathcal{R}_{f}$, where $i:\left(\mathbf{R}^{2}, 0\right) \rightarrow\left(\mathbf{R}^{3}, 0\right), i(x, y)=(x, y, 0)$, and we see that $g$ is not an extendable unfolding of $f$.

The versal opening of $g$ is given by $G:\left(\mathbf{R}^{3}, 0\right) \rightarrow\left(\mathbf{R}^{7}, 0\right)=\left(\mathbf{R}^{3} \times \mathbf{R}^{4}, 0\right)$,

$$
\begin{aligned}
& G(x, y, u)=\left(g(x, y, u), x^{3}+y^{3}+3 x y u, x^{5}+5 x^{3} y u-12 x^{2} u^{3}+9 y u^{4},\right. \\
& \left.y^{5}+5 x y^{3} u-12 y^{2} u^{3}+9 x u^{4}, x^{3} y^{3}-12 x^{2} y^{2} u^{2}-11 x^{3} u^{3}-11 y^{3} u^{3}-12 x y u^{4}\right) .
\end{aligned}
$$

Then

$$
G(x, y, 0)=\left(\frac{1}{2} x^{2}, \frac{1}{2} y^{2}, x^{3}+y^{3}, x^{5}, y^{5}, x^{3} y^{3}\right)
$$

is not a versal opening of $f=\left(\frac{1}{2} x^{2}, \frac{1}{2} y^{2}\right)$. Note that the element $\psi_{3}$ gives a Lagrange immersion of type $D_{4}^{+}$, which is a Lagrange stable lifting of $g$. Other elements are obtained by operating lowerable vector fields of $g$ to $\psi_{3}$. See $\S 8$. 
Example 7.3 (Hyperbolic case.) Let $h:\left(\mathbf{R}^{4}, 0\right) \rightarrow\left(\mathbf{R}^{4}, 0\right)$ be the stable map-germ

$$
h(x, y, \lambda, \mu)=(z, w, \lambda, \mu)=\left(\frac{1}{2} x^{2}+y \lambda, \frac{1}{2} y^{2}+x \mu, \lambda, \mu\right) .
$$

of $\mathcal{K}$-class $I_{2,2}([20])$. Then $\mathcal{R}_{f}$ is minimally generated over $h^{*} \mathcal{E}_{\mathbf{R}^{4}, 0}$ by 1 and

$$
\begin{aligned}
\varphi_{4} & =x^{3} \mu+y^{3} \lambda+3 x y \lambda \mu \\
\varphi_{5}^{3,2} & =x^{3} y^{2}-2 x^{2} y \lambda \mu+x \lambda^{2} \mu^{2} \\
\varphi_{5}^{2,3} & =x^{2} y^{3}-2 x y^{2} \lambda \mu+y \lambda^{2} \mu^{2} \\
\varphi_{5}^{5,0} & =x^{5}+5 x^{3} y \lambda+15 y \lambda^{3} \mu \\
\varphi_{5}^{0,5} & =y^{5}+5 x y^{3} \mu+15 x \lambda \mu^{3} \\
\varphi_{6} & =x^{3} y^{3}-3 x y \lambda^{2} \mu^{2} .
\end{aligned}
$$

We have the mini-versal opening $H:\left(\mathbf{R}^{4}, 0\right) \rightarrow\left(\mathbf{R}^{4} \times \mathbf{R}^{6}, 0\right)=\left(\mathbf{R}^{10}, 0\right)$ of $h$ by

$$
H=\left(f, \varphi_{4}, \varphi_{5}^{3,2}, \varphi_{5}^{2,3}, \varphi_{5}^{5,0}, \varphi_{5}^{0,5}, \varphi_{6}\right) .
$$

Moreover we see that

$$
j^{*} \mathcal{R}_{h} \subsetneq \mathcal{R}_{g}\left(\subsetneq \mathcal{E}_{\mathbf{R}^{3}, 0}\right), \quad(j \circ i)^{*} \mathcal{R}_{h} \subsetneq i^{*} \mathcal{R}_{g} \subsetneq \mathcal{R}_{f}\left(\subsetneq \mathcal{E}_{\mathbf{R}^{3}, 0}\right),
$$

where $j:\left(\mathbf{R}^{3}, 0\right) \rightarrow\left(\mathbf{R}^{4}, 0\right), j(x, y, u)=(x, y, u, u)$. Thus the unfolding $h$ of $f$ is not extendable, which is also not extendable regarded as an unfolding of $g$ as well.

Now we show the concrete way of calculations for Example 7.3 to make sure ourselves:

Let $k \in \mathcal{E}_{\mathbf{R}^{4}, 0}=\mathcal{E}_{4}$. By the preparation theorem, we set,

$$
k=\left(a_{0} \circ h\right)+\left(a_{1} \circ h\right) x+\left(a_{2} \circ h\right) y+\left(a_{3} \circ h\right) x y .
$$

Then

$$
d k \equiv\left(\left(a_{1} \circ h\right)+\left(a_{3} \circ h\right) y\right) d x+\left(\left(a_{2} \circ h\right)+\left(a_{3} \circ h\right) x\right) d y\left(\bmod . \mathcal{J}_{h}\right) .
$$

We suppose $d k$ is equal to the form

$$
\begin{aligned}
A d z & +B d w+C d \lambda+D d \mu \\
& =(A x+B \mu) d x+(A \lambda+B y) d y+(A y+C) d \lambda+(B x+D) d \mu .
\end{aligned}
$$


Then we have

$$
\begin{gathered}
\left(a_{1} \circ h\right)+\left(a_{3} \circ h\right) y=A x+B \mu,\left(a_{2} \circ h\right)+\left(a_{3} \circ h\right) x=A \lambda+B y, \\
A y+C=0, B x+D=0 .
\end{gathered}
$$

Then $C=-A y, D=-B x$. Now set

$$
\begin{aligned}
& A=\left(A_{0} \circ h\right)+\left(A_{1} \circ h\right) x+\left(A_{2} \circ h\right) y+\left(A_{3} \circ h\right) x y, \\
& B=\left(B_{0} \circ h\right)+\left(B_{1} \circ h\right) x+\left(B_{2} \circ h\right) y+\left(B_{3} \circ h\right) x y .
\end{aligned}
$$

Then we have

$$
A x=\left(2 z A_{1}-4 w A_{3}\right)+\left(A_{0}+4 \lambda \mu A_{3}\right) x+\left(-2 \lambda A_{1}+2 z A_{3}\right) y+A_{2} x y,
$$

omitting "oh". Similarly we have

$$
\begin{aligned}
& B y=\left(2 w B_{2}-4 z \mu B_{3}\right)+\left(-2 \mu B_{2}+2 w B_{3}\right) x+\left(B_{0}+4 \lambda \mu B_{3}\right) y+B_{1} x y, \\
& A \lambda=\left(\lambda A_{0}\right)+\left(\lambda A_{1}\right) x+\left(\lambda A_{2}\right) y+\left(\lambda A_{3}\right) x y . \\
& B \mu=\left(\mu B_{0}\right)+\left(\mu B_{1}\right) x+\left(\mu B_{2}\right) y+\left(\mu B_{3}\right) x y .
\end{aligned}
$$

Then we set, to find analytic or formal generators,

$$
\begin{gathered}
a_{1}=2 z A_{1}-4 w \lambda A_{3}+\mu B_{0}, \quad a_{3}=-2 \lambda A_{1}+2 z A_{3}+\mu B_{2}, \\
0=A_{0}+4 \lambda \mu A_{3}+\mu B_{1}, \quad 0=A_{2}+\mu B_{3},
\end{gathered}
$$

and

$$
\begin{gathered}
a_{2}=2 w B_{2}-4 z \mu B_{3}+\lambda A_{0}, \quad a_{3}=-2 \mu B_{2}+2 w B_{3}+\lambda A_{1}, \\
0=B_{0}+4 \lambda \mu B_{3}+\lambda A_{2}, \quad 0=B_{1}+\lambda A_{3} .
\end{gathered}
$$

Then we are led to the relation:

$$
3 \lambda A_{1}-3 \mu B_{2}-2 z A_{3}+2 w B_{3}=0 \cdots \cdots \cdots(*) .
$$

If $A_{1}, A_{2}, B_{2}, B_{3}$ satisfy the relation (*), then $A_{0}, A_{2}, B_{0}, B_{1}$ are determined from them and so $a_{1} \circ h, a_{2} \circ h, a_{3} \circ h$ :

$$
A_{0}=-3 \lambda \mu A_{3}, A_{2}=-\mu B_{3}, B_{0}=-3 \lambda \mu B_{3}, B_{1}=-\lambda A_{3},
$$

and

$$
\begin{aligned}
& a_{1}=2 z A_{1}-4 w \lambda A_{3}-3 \lambda \mu^{2} B_{3}, \\
& a_{2}=-3 \lambda^{2} \mu A_{3}+2 w B_{2}-4 z \mu B_{3}, \\
& a_{3}=-2 \lambda A_{1}+2 z A_{3}+\mu B_{2}\left(=\lambda A_{1}-2 \mu B_{2}+2 w B_{3}\right) .
\end{aligned}
$$


Since $\left(3 \lambda,-3 \mu,-2 x, 2 w\right.$ are regular sequence in $\mathcal{E}_{4}$, the first Koszul cohomology for them vanishes (see for instance, [21]). Then, by setting $\left(A_{1}, B_{2}, A_{3}, B_{3}\right)$ as

$$
\begin{gathered}
(\mu, \lambda, 0,0),(2 z, 0,3 \lambda, 0),(2 w, 0,0,-3 \lambda), \\
(0,2 z,-3 \mu, 0),(0,2 w, 0,3 \mu),(0,0, w, z),
\end{gathered}
$$

respectively, we have elements $\varphi_{4}, \varphi_{5}^{3,2}, \varphi_{5}^{2,3}, \varphi_{5}^{5,0}, \varphi_{5}^{0,5}, \varphi_{6}$. such that

$$
1, \varphi_{4}, \varphi_{5}^{3,2}, \varphi_{5}^{2,3}, \varphi_{5}^{5,0}, \varphi_{5}^{0,5}, \varphi_{6}
$$

generate $\mathcal{R}_{h}^{\omega}$ over $h^{*} \mathcal{O}_{4}$. Then, by Proposition 6.2 , they generate $\mathcal{R}_{h}$ over $h^{*} \mathcal{E}_{4}$.

Similarly we have the following.

Example 7.4 (Elliptic case.) Let $k:\left(\mathbf{R}^{4}, 0\right) \rightarrow\left(\mathbf{R}^{4}, 0\right)$ be the stable mapgerm given by

$$
k(x, y, \lambda, \mu)=\left(\frac{1}{2}\left(x^{2}-y^{2}\right)+\lambda x+\mu y, x y+\mu x-\lambda y, \lambda, \mu\right),
$$

of $\mathcal{K}$-class $I I_{2,2}$. Then $\mathcal{R}_{k}$ is minimally generated over $k^{*} \mathcal{E}_{4}$ by 1 and $\rho_{4}, \rho_{5}^{3,2}, \rho_{5}^{2,3}, \rho_{5}^{5,0}, \rho_{5}^{0,5}, \rho_{6}$, where

$$
\begin{aligned}
\rho= & a_{1} x+a_{2} y+\frac{1}{2} a_{3}\left(x^{2}+y^{2}\right), \\
a_{1}= & 2 z A_{1}+2 w A_{2}+\left(-\frac{3}{2} \lambda^{3}-\frac{3}{2} \lambda \mu^{2}-3 z \lambda-3 w \mu\right) A_{3} \\
& +\left(-\frac{3}{2} \lambda^{2} \mu-\frac{3}{2} \mu^{3}-3 z \mu+3 w \lambda\right) B_{3}, \\
a_{2}= & -2 w A_{1}+2 z A_{2}+\left(\frac{3}{2} \lambda^{2} \mu-\frac{3}{2} \mu^{3}+z \mu-w \lambda\right) A_{3}, \\
& +\left(\frac{3}{2} \lambda^{3}+\frac{3}{2} \lambda \mu^{2}-z \lambda-3 w \mu\right) B_{3}, \\
a_{3}= & -\lambda A_{1}-\mu A_{2}+\left(z-\frac{1}{2} \lambda^{2}+\frac{1}{2} \mu^{2}\right) A_{3}+(w-\lambda \mu) B_{3},
\end{aligned}
$$

and $\rho=\rho_{4}, \rho_{5}^{3,2}, \rho_{5}^{2,3}, \rho_{5}^{5,0}, \rho_{5}^{0,5}, \rho_{6}$ respectively for

$$
\begin{aligned}
\left(A_{1}, A_{2}, A_{3}, B_{3}\right)= & (\lambda, \mu, 0,0), \\
& \left(0, z-\frac{3}{2} \lambda^{2}+\frac{3}{2} \mu^{2}, 0,3 \lambda\right), \\
& (0, w-3 \lambda \mu,-3 \lambda, 0), \\
& \left(z-\frac{3}{2} \lambda^{2}+\frac{3}{2} \mu^{2}, 0,0,-3 \mu\right), \\
& (w-3 \lambda \mu, 0,3 \mu, 0), \\
& \left.\left(0,0, z-\frac{3}{2} \lambda^{2}+\frac{3}{2} \mu^{2}, w-3 \lambda \mu\right)\right) .
\end{aligned}
$$

Note that, in the process of calculations, we see that $A_{1}, A_{2}, A_{3}, B_{3}$ obey the relation

$$
(3 \mu) A_{1}+(-3 \lambda) A_{2}+(-w+3 \lambda \mu) A_{3}+\left(z-\frac{3}{2} \lambda^{2}+\frac{3}{2} \mu^{2}\right) B_{3}=0 .
$$

and that $3 \mu,-3 \lambda,-w+3 \lambda \mu, z-\frac{3}{2} \lambda^{2}+\frac{3}{2} \mu^{2}$ form a regular sequence in $\mathcal{E}_{4}$. 
Theorem 7.5 Any stable mono-germ $\left(\mathbf{R}^{4}, a\right) \rightarrow\left(\mathbf{R}^{4}, b\right)$, and therefore any stable multi-germ $\left(\mathbf{R}^{4}, A\right) \rightarrow\left(\mathbf{R}^{4}, b\right)$ has a versal opening.

Proof: Let $f:\left(\mathbf{R}^{4}, a\right) \rightarrow\left(\mathbf{R}^{4}, b\right)$ be a stable map-germ. Then $f$ is of corank $\leq 1$ and is diffeomorphic to a Morin map or $f$ is of corank 2 and is diffeomorphic to the germ $h$ of Example 7.3 or $k$ of Example 7.4 (see for instance [7]). In the case of corank one, we have constructed the versal opening in Proposition 5.1. In the case of corank two, we have constructed the versal opening, using a normal form of $f$ in Examples 7.3 and Example 7.4. If a map-germ $\ell$ has a versal opening and $f$ is diffeomorphic to $\ell$, then $f$ has a versal opening (cf. Lemma $2.2(3)$ ). Therefore $f$ has a versal opening. Let $f:\left(\mathbf{R}^{4}, A\right) \rightarrow\left(\mathbf{R}^{4}, b\right)$ be a stable multi-germ. Then, for each $a_{i} \in A$, the restriction $f_{i}:\left(\mathbf{R}^{4}, a_{i}\right) \rightarrow\left(\mathbf{R}^{4}, b\right)$ of $f$ to $\left(\mathbf{R}^{n}, a_{i}\right)$ is a stable germ. Since each $f_{i}$ has a versal opening, we see that $f$ itself has a versal opening by Remark 2.8.

\section{Openings and lowerable vector fields}

Let $f:\left(\mathbf{R}^{n}, A\right) \rightarrow\left(\mathbf{R}^{m}, b\right)$ a map-germ. A germ of vector field $\xi$ over $\left(\mathbf{R}^{n}, A\right)$ is called lowerable for $f$, or $f$-lowerable, if there exists a germ of vector field $\eta$ over $\left(\mathbf{R}^{m}, b\right)$ such that $f_{*} \xi=\eta \circ f$ as a germ of vector field along $f$. The lowerable vector fields form an $f^{*} \mathcal{E}_{\mathbf{R}^{m}, b}$-module, which is denoted by $\mathfrak{X}_{f}$.

Lemma 8.1 (cf. [13]) Let $f:\left(\mathbf{R}^{n}, A\right) \rightarrow\left(\mathbf{R}^{m}, b\right)$ be a map-germ and $\xi$ a lowerable vector field. Then $\xi\left(f^{*} \mathcal{E}_{\mathbf{R}^{m}, b}\right) \subseteq f^{*} \mathcal{E}_{\mathbf{R}^{m}, b}$ and $\xi\left(\mathcal{R}_{f}\right) \subseteq \mathcal{R}_{f}$. Therefore $\mathfrak{X}_{f}\left(f^{*} \mathcal{E}_{\mathbf{R}^{m}, b}\right) \subseteq f^{*} \mathcal{E}_{\mathbf{R}^{m}, b}$ and $\mathfrak{X}_{f}\left(\mathcal{R}_{f}\right) \subseteq \mathcal{R}_{f}$.

Proof: Suppose $f_{*} \xi=\eta \circ f$ as in the above definition.

Let $a \in f^{*} \mathcal{E}_{\mathbf{R}^{m}, b}$. Then

$$
\begin{aligned}
\xi\left(f^{*} a\right)(x) & =\left\langle d\left(f^{*} a\right)(x), \xi(x)\right\rangle=\left\langle f^{*}(d a)(x), \xi(x)\right\rangle=\left\langle(d a)(f(x)), f_{*}(\xi(x))\right\rangle \\
& =\langle(d a)(f(x)), \eta(f(x))\rangle=(\eta a)(f(x))=\left(f^{*}(\eta a)\right)(x) .
\end{aligned}
$$

Therefore $\xi\left(f^{*} a\right)=f^{*}(\eta a)$.

Let $b \in \mathcal{R}_{f}$. Then $d b=\sum_{i=1}^{m} p_{i} d f_{i}$ for some $p_{i} \in \mathcal{E}_{\mathbf{R}^{n}, A}$. Then we have

$$
d(\xi b)=L_{\xi}\left(\sum_{i=1}^{m} p_{i} d f_{i}\right)=\sum_{i=1}^{m}\left(\xi p_{i}\right) d f_{i}+\sum_{i=1}^{m} p_{i} d\left(\xi f_{i}\right) .
$$

Since each $\xi f_{i} \in f^{*} \mathcal{E}_{\mathbf{R}^{m}, b} \subseteq \mathcal{R}_{f}$, we see $d(\xi b) \in \mathcal{J}_{f}$. Therefore $\xi b \in \mathcal{R}_{f}$. 
The differential operator $D: \mathcal{E}_{\mathbf{R}^{n}, A} \rightarrow \mathcal{E}_{\mathbf{R}^{n}, A}$ is called lowerable for $f$ : $\left(\mathbf{R}^{n}, A\right) \rightarrow\left(\mathbf{R}^{m}, b\right)$, if $D$ is a finite sum of operators of form

$$
\xi_{1} \xi_{2} \cdots \xi_{s}
$$

with coefficients in $f^{*} \mathcal{E}_{\mathbf{R}^{n}, b}$, where $\xi_{1}, \xi_{2}, \ldots, \xi_{s}$ are lowerable vector field (regarded as first order differential operators) for $f$. The lowerable differential operators form a (non-commutative) $f^{*} \mathcal{E}_{\mathbf{R}^{n}, b}$-algebra, which is denoted by $\mathfrak{L}_{f}$.

By Lemma 8.1, we have:

Corollary 8.2 Let $f:\left(\mathbf{R}^{n}, A\right) \rightarrow\left(\mathbf{R}^{m}, b\right)$ be a map-germ and $D: \mathcal{E}_{\mathbf{R}^{n}, A} \rightarrow$ $\mathcal{E}_{\mathbf{R}^{n}, A}$ be a lowerable differential operator. Then $D\left(f^{*} \mathcal{E}_{\mathbf{R}^{m}, b}\right) \subseteq f^{*} \mathcal{E}_{\mathbf{R}^{m}, b}$ and $D\left(\mathcal{R}_{f}\right) \subseteq \mathcal{R}_{f}$. Therefore $\mathfrak{L}_{f}\left(f^{*} \mathcal{E}_{\mathbf{R}^{m}, b}\right) \subseteq f^{*} \mathcal{E}_{\mathbf{R}^{m}, b}$ and $\mathfrak{L}_{f}\left(\mathcal{R}_{f}\right) \subseteq \mathcal{R}_{f}$.

We conclude the present paper by examining how lowerable vector fields acts on the ramification modules and the structure of mini-versal openings for Example 7.1, Example 7.2 and Example 7.3.

Example 8.3 The lowerable vector fields for $f$ in Example 7.1 is generated by

$$
\xi_{1}=x \frac{\partial}{\partial x}, \quad \xi_{2}=y \frac{\partial}{\partial y}
$$

over $f^{*} \mathcal{E}_{2}$. Then we have

$$
\begin{gathered}
\xi_{1}\left(x^{3}\right)=3 x^{3}, \quad \xi_{2}\left(x^{3}\right)=0, \\
\xi_{1}\left(y^{3}\right)=0, \quad \xi_{2}\left(y^{3}\right)=3 y^{3}, \\
\xi_{1}\left(x^{3} y^{3}\right)=3 x^{3} y^{3}, \quad \xi_{2}\left(x^{3} y^{3}\right)=3 x^{3} y^{3} .
\end{gathered}
$$

Define the module $S=\left\langle 1, x^{3}, y^{3}\right\rangle_{f^{*} \mathcal{E}_{m}} \subsetneq \mathcal{R}_{f}$ over $f^{*} \mathcal{E}_{m}$. Then we see

$$
\mathfrak{X}_{f}(S)=S, \quad \mathfrak{L}_{f}(S)=S .
$$

The lowerable vector fields for $g$ in Example 7.2 is generated by

$$
\left\{\begin{array}{l}
\xi_{0}=x \frac{\partial}{\partial x}+y \frac{\partial}{\partial y}+u \frac{\partial}{\partial u}, \\
\xi_{1}=\left(-\frac{3}{2} u^{3}+\frac{1}{3} z x\right) \frac{\partial}{\partial x}+\left(-\frac{1}{2} u^{2} x\right) \frac{\partial}{\partial y}+\left(-\frac{1}{3} z u\right) \frac{\partial}{\partial u}, \\
\xi_{2}=\left(\frac{1}{3} w x+\frac{1}{2} u^{2} y\right) \frac{\partial}{\partial x}+\left(\frac{3}{2} u^{3}-\frac{1}{2} u x y\right) \frac{\partial}{\partial y}+\frac{2}{3} w u \frac{\partial}{\partial u}, \\
\xi_{3}=\left(-\frac{3}{2} w u^{2}-\frac{1}{2} z u y+\frac{1}{2} w x y\right) \frac{\partial}{\partial x}+\left(\frac{3}{2} u^{3}+\frac{1}{2} z x y\right) \frac{\partial}{\partial y}+(-z w) \frac{\partial}{\partial u},
\end{array}\right.
$$


over $g^{*} \mathcal{E}_{2}$, where $z=\frac{1}{2} x^{2}+u y, w=\frac{1}{2} y^{2}+u x$. Then we have that

\section{$1, \xi_{0} \psi_{3}, \xi_{1} \psi_{3}, \xi_{2} \psi_{3}, \xi_{3} \psi_{3}$}

generate $\mathcal{R}_{g}$ over $g^{*} \mathcal{E}_{3}$. This fact is a consequence of Lagrange stability of induced Lagrangian immersion from $\left(g, \psi_{3}\right), \widetilde{g}:\left(\mathbf{R}^{3}, 0\right) \rightarrow T^{*} \mathbf{R}^{3}=\mathbf{R}^{6}$ defined by

$\widetilde{g}=\left(z, w, u, p_{1}, p_{2}, p_{3}\right)=\left(z=\frac{1}{2} x^{2}+u y, w=\frac{1}{2} y^{2}+u x, u, 3 x, 3 y,-3 x y\right)$.

See [10]. Therefore, if we consider the $g^{*} \mathcal{E}_{3}$-module $T$ generated by $1, \psi_{3}$, then we have that

$$
\mathfrak{X}_{g}(T)=\mathcal{R}_{g}, \quad \mathfrak{L}_{g}(T)=\mathcal{R}_{g}
$$

The lowerable vector fields for $g$ in Example 7.3 is generated by

$$
\left\{\begin{aligned}
\xi_{0} & =x \frac{\partial}{\partial x}+y \frac{\partial}{\partial y}+\lambda \frac{\partial}{\partial \lambda}+\mu \frac{\partial}{\partial \mu} \\
\xi_{1} & =x \frac{\partial}{\partial x}-y \frac{\partial}{\partial y}+(3 \lambda) \frac{\partial}{\partial \lambda}+(-3 \mu) \frac{\partial}{\partial \mu} \\
\xi_{2} & =(-3 \lambda \mu+x y) \frac{\partial}{\partial x}+(-\mu x) \frac{\partial}{\partial y}+\left(-x^{2}-2 \lambda y\right) \frac{\partial}{\partial \lambda} \\
\xi_{3} & =(-\lambda y) \frac{\partial}{\partial x}+(-3 \lambda \mu+x y) \frac{\partial}{\partial y}+\left(-y^{2}-2 \mu x\right) \frac{\partial}{\partial \mu}
\end{aligned}\right.
$$

Then we have $\xi_{0} \varphi_{4}=4 \varphi_{4}, \xi_{1} \varphi_{4}=0$ and

$$
\begin{aligned}
& \xi_{2} \varphi_{4}=-\varphi_{5}^{2,3}-8 \lambda\left(w^{2}+z \mu^{2}\right) \equiv-\varphi_{5}^{2,3}\left(\bmod . h^{*} \mathcal{E}_{4}\right), \\
& \xi_{3} \varphi_{4}=-\varphi_{5}^{3,2}-8 \mu\left(z^{2}+w \lambda^{2}\right) \equiv-\varphi_{5}^{3,2}\left(\bmod . h^{*} \mathcal{E}_{4}\right) .
\end{aligned}
$$

Moreover

$$
\begin{gathered}
\xi_{0} \varphi_{5}^{3,2}=5 \varphi_{5}^{3,2}, \xi_{0} \varphi_{5}^{2,3}=5 \varphi_{5}^{2,3}, \xi_{0} \varphi_{5}^{5,0}=5 \varphi_{5}^{5,0}, \xi_{0} \varphi_{5}^{0,5}=5 \varphi_{5}^{0,5}, \\
\xi_{1} \varphi_{5}^{3,2}=\varphi_{5}^{3,2}, \xi_{1} \varphi_{5}^{2,3}=-\varphi_{5}^{2,3}, \xi_{1} \varphi_{5}^{5,0}=5 \varphi_{5}^{5,0}, \xi_{1} \varphi_{5}^{0,5}=-5 \varphi_{5}^{0,5},
\end{gathered}
$$


and

$$
\begin{aligned}
\xi_{2} \varphi_{5}^{3,2} & =3 \varphi_{6}^{3,3}+18 \lambda \mu \varphi_{4}-36 z w \lambda \mu-3 \lambda^{3} \mu^{3} \\
& \equiv 3 \varphi_{6}^{3,3}+18 \lambda \mu \varphi_{4}\left(\bmod . h^{*} \mathcal{E}_{4}\right), \\
\xi_{2} \varphi_{5}^{2,3} & =-4 \lambda \varphi_{5}^{0,5}-32 z^{2} \mu^{2}+68 w \lambda^{2} \mu^{2} \\
& \equiv-4 \lambda \varphi_{5}^{0,5}\left(\bmod . h^{*} \mathcal{E}_{4}\right), \\
\xi_{2} \varphi_{5}^{5,0} & =5 \lambda \varphi_{5}^{3,2}-16 z^{2} \mu-4 w \lambda^{2} \mu \\
& \equiv 5 \lambda \varphi_{5}^{3,2}\left(\bmod . h^{*} \mathcal{E}_{4}\right), \\
\xi_{2} \varphi_{5}^{0,5} & =15 \mu^{2} \varphi_{4}-60 z w \mu^{2}-45 \lambda^{2} \mu^{4} \\
& \equiv 15 \mu^{2} \varphi_{4}\left(\bmod . h^{*} \mathcal{E}_{4}\right), \\
\xi_{3} \varphi_{5}^{3,2} & =-4 \mu \varphi_{5}^{5,0}-32 w^{2} \lambda^{2}+68 z \lambda^{3} \mu^{2} \\
& \equiv-4 \mu \varphi_{5}^{5,0}\left(\bmod . h^{*} \mathcal{E}_{4}\right), \\
\xi_{3} \varphi_{5}^{2,3} & =3 \varphi_{6}^{3,3}+18 \lambda \mu \varphi_{4}-36 z w \lambda \mu-3 \lambda^{3} \mu^{2} \\
& =3 \varphi_{6}^{3,3}+18 \lambda \mu \varphi_{4}\left(\bmod . h^{*} \mathcal{E}_{4}\right), \\
\xi_{3} \varphi_{5}^{5,0} & =15 \lambda^{2} \varphi_{4}-60 z w \lambda^{2}-45 \lambda^{4} \mu^{2} \\
& \equiv 15 \lambda^{2} \varphi_{4}\left(\bmod . h^{*} \mathcal{E}_{4}\right), \\
\xi_{3} \varphi_{5}^{0,5} & =5 \mu \varphi_{5}^{2,3}-16 w^{2} \lambda-4 z \lambda \mu^{2} \\
& \equiv 5 \mu \varphi_{5}^{2,3}\left(\bmod . h^{*} \mathcal{E}_{4}\right) .
\end{aligned}
$$

We consider the the $h^{*} \mathcal{E}_{4}$-module $U$ generated by $1, \varphi_{4}$ and $V$ generated by $1, \varphi_{4}, \varphi_{5}^{3,2}, \varphi_{5}^{2,3}, \varphi_{5}^{5,0}, \varphi_{5}^{0,5}$. Then we have

$$
\mathfrak{X}_{h}(U)=V, \quad \mathfrak{L}_{h}(U)=\mathcal{R}_{h} .
$$

\section{References}

[1] V.I. Arnol'd, Catastrophe theory, 3rd edition, Springer-Verlag, (1992).

[2] E. Bierstone, P.D. Milman, Relations among analytic functions I, Ann. Inst. Fourier, Grenoble, 37-1 (1987), 187-239.

[3] E. Bierstone, P.D. Milman, Relations among analytic functions II, Ann. Inst. Fourier, Grenoble, 37-2 (1987), 49-77.

[4] T. Bröcker, Differentiable Germs and Catastrophes, London Math. Soc. Lecture Note Series 17, Cambridge Univ. Press (1975).

[5] J.W. Bruce, P.J. Giblin, Curves and singularities, A geometrical introduction to singularity theory, 2nd ed., Cambridge Univ. Press, (1992). 
[6] T. Gaffney, M.A. Vitulli, Weak subintegral closure of ideals, Arxiv 0708.3105v2.

[7] M. Golubitsky, V. Guillemin, Stable mappings and their singularities, Graduate Texts in Math., Springer-Verlag (1974).

[8] A.B. Givental', Whitney singularities of solutions of partial differential equations, J. Geom. Phys. 15-4 (1995) 353-368.

[9] G. Ishikawa, Families of functions dominated by distributions of $\mathcal{C}$-classes of mappings, Ann. Inst. Fourier, 33-2 (1983), 199-217.

[10] G. Ishikawa, Lagrangian stability of a Lagrangian map-germ in a restricted class - open Whitney umbrellas and open swallowtails, Proceedings of the Symposium "Singularity Theory and its Applications", 1989, Hokkaido Univ., ed. by G. Ishikawa, S. Izumiya and T. Suwa, Hokkaido Univ. Technical Report Series in Math., 12 (1989), $267-273$

[11] G. Ishikawa, Parametrization of a singular Lagrangian variety, Trans. Amer. Math. Soc., 331-2 (1992), 787-798.

[12] G. Ishikawa, Parametrized Legendre and Lagrange varieties, Kodai Math. J., 17-3 (1994), 442-451.

[13] G. Ishikawa, Developable of a curve and determinacy relative to osculation-type, Quart. J. Math. Oxford, 46 (1995).

[14] G. Ishikawa, Symplectic and Lagrange stabilities of open Whitney umbrellas, Invent. math., bf 126 (1996), 215-234.

[15] G. Ishikawa, Singularities of tangent varieties to curves and surfaces, Arxiv. math.DG.1201.2778, to appear in Journal of Singularities.

[16] G. Ishikawa, Tangent varieties and openings of map-germs, preprint (submitted).

[17] G. Ishikawa, S. Janeczko, Symplectic bifurcations of plane curves and isotropic liftings, Quarterly J. Math. Oxford, 54 (2003), 73-102.

[18] B. Malgrange, Ideals of Differentiable Functions, Oxford Univ. Press (1966).

[19] J. Martinet, Singularities of Smooth Functions and Maps, London Math. Soc. Lecture Note Series 58, Cambridge Univ. Press (1982).

[20] J.N. Mather, Stability of $C^{\infty}$ mappings IV: The nice dimensions, Lecture Notes in Math. 192, Springer (1971), pp.192-253.

[21] H. Matsumura, Commutative Algebra, Benjamin (1970).

[22] D. Mond, Deformations which preserve the non-immersive locus of a map-germ, Math. Scand., 66 (1990), 21-32.

[23] C.T.C. Wall, Finite determinacy of smooth map-germs, Bull. London Math. Soc. 13 (1981), 481-539.

Acknowledgement: This paper has the origin in my master thesis [9]. From the time when I was undergraduate student of Kyoto University under the supervision of Professor Masahisa Adachi, Professor Satoshi Koike keeps to give me kind encouragements and friendship throughout. I would like to thank him in the occasion of his 60th birthday. 
Goo Ishikawa

Department of Mathematics, Hokkaido University, Japan

e-mail: ishikawa@math.sci.hokudai.ac.jp 